Article

\title{
A Survey in Natural Forest Ecosystems of Vietnam Reveals High Diversity of both New and Described Phytophthora Taxa including $P$. ramorum
}

\author{
Thomas Jung ${ }^{1,2, *(\mathbb{D})}$, Bruno Scanu $^{3}{ }^{(\mathbb{D}}$, Clive M. Brasier $^{4}$, Joan Webber ${ }^{4}$, Ivan Milenković ${ }^{1}{ }^{(\mathbb{D}}$, \\ Tamara Corcobado ${ }^{1}$, Michal Tomšovský ${ }^{1}{ }^{\circledR}$, Matěj Pánek ${ }^{1,5}$, József Bakonyi ${ }^{6}$, Cristiana Maia ${ }^{7}$, \\ Aneta Bačová ${ }^{1}$, Milica Raco ${ }^{1}$, Helen Rees ${ }^{4,8}$, Ana Pérez-Sierra ${ }^{4}$ and Marília Horta Jung ${ }^{1,2}$ (1) \\ 1 Phytophthora Research Centre, Mendel University in Brno, 61300 Brno, Czech Republic; \\ ivan.milenkovic@mendelu.cz (I.M.); tamara.sanchez@mendelu.cz (T.C.); tomsovsk@mendelu.cz (M.T.); \\ panek@vurv.cz (M.P.); aneta.bacova@mendelu.cz (A.B.); milica.raco@mendelu.cz (M.R.); \\ marilia.jung@mendelu.cz (M.H.J.) \\ 2 Phytophthora Research and Consultancy, Am Rain 9, 83131 Nußdorf, Germany \\ 3 Dipartimento di Agraria, Sezione di Patologia vegetale ed Entomologia (SPaVE), Università degli Studi di \\ Sassari, Viale Italia 39, 07100 Sassari, Italy; bscanu@uniss.it \\ 4 Forest Research, Alice Holt Lodge, Farnham, Surrey GU10 4LH, UK; \\ clive.brasier@forestresearch.gov.uk (C.M.B.); joan.webber@forestresearch.gov.uk (J.W.); \\ helen.rees@bristol.ac.uk (H.R.); ana.perez-sierra@forestresearch.gov.uk (A.P.-S.) \\ 5 Crop Research Institute, Drnovská 507/73, 16106 Prague 6, Czech Republic \\ 6 Plant Protection Institute, Centre for Agricultural Research, Herman Ottó út 15, 1022 Budapest, Hungary; \\ bakonyi.jozsef@agrar.mta.hu \\ 7 Centre of Marine Sciences (CCMAR), University of Algarve, 8005-139 Faro, Portugal; ccmaia@ualg.pt \\ 8 School of Life Sciences, University of Bristol, 24 Tyndall Avenue, Bristol BS8 1TQ, UK \\ * Correspondence: thomas.jung@mendelu.cz; Tel.: +420-5451-361-72
}

Received: 22 December 2019; Accepted: 10 January 2020; Published: 12 January 2020

\begin{abstract}
In 2016 and 2017, surveys of Phytophthora diversity were performed in 25 natural and semi-natural forest stands and 16 rivers in temperate and subtropical montane and tropical lowland regions of Vietnam. Using baiting assays from soil samples and rivers and direct isolations from naturally fallen leaves, 13 described species, five informally designated taxa and 21 previously unknown taxa of Phytophthora were isolated from 58 of the 91 soil samples $(63.7 \%)$ taken from the rhizosphere of 52 of the 64 woody plant species sampled (81.3\%) in 20 forest stands $(83.7 \%)$, and from all rivers: P. capensis, P. citricola VII, VIII, IX, X and XI, P. sp. botryosa-like 2, P. sp. meadii-like 1 and 2, $P$. sp. tropicalis-like 2 and $P$. sp. multivesiculata-like 1 from Phytophthora major phylogenetic Clade 2; $P$. castaneae and $P$. heveae from Clade 5; P. chlamydospora, P. gregata, $P$. sp. bitahaiensis-like and $P$. sp. sylvatica-like 1, 2 and 3 from Clade 6; P. cinnamomi $\left(P_{c}\right)$, P. parvispora, P. attenuata, $P$. sp. attenuata-like 1,2 and 3 and P. Xheterohybrida from Clade 7; P. drechsleri, P. pseudocryptogea, P. ramorum (Pr) and P. sp. kelmania from Clade 8, $P$. macrochlamydospora, $P$. sp. Xinsolita-like, $P$. sp. $\times$ kunnunara-like, $P$. sp. $\times$ virginiana-like s.l. and three new taxa, $P$. sp. quininea-like, $P$. sp. $\times$ Grenada 3-like and $P$. sp. $\times$ Peru 4-like, from Clade 9; and P. sp. gallica-like 1 and 2 from Clade 10. The A1 and A2 mating types of both $P c$ and $P r$ co-occurred. The A2 mating type of $P c$ was associated with severe dieback of montane forests in northern Vietnam. Most other Phytophthora species, including $P r$, were not associated with obvious disease symptoms. It is concluded that (1) Vietnam is within the center of origin of most Phytophthora taxa found including $P c$ and $P r$, and (2) Phytophthora clades 2, 5, 6, 7, 8, 9, and 10 are native to Indochina.
\end{abstract}

Keywords: biosecurity; breeding systems; hybridization; Phytophthora cinnamomi; biogeography; center of origin 


\section{Introduction}

The number of devastating declines of trees and other woody plants driven by introduced invasive Phytophthora species in natural ecosystems in Australia, Europe, and North America has increased exponentially since the 1960s [1-9]. Therefore, numerous surveys in natural and semi-natural ecosystems have been performed in the past two decades to assess Phytophthora diversity in these continents and in Africa, Asia, and South America [4,5,10-19]. As a result of these surveys and molecular re-evaluations of culture collections and several species complexes, the number of described species and informally designated taxa of Phytophthora has tripled since 1999 [2,18,20-28]. A conservative estimate predicted the existence of 200-600 unknown Phytophthora species in natural ecosystems of as yet unsurveyed regions of the world [26]. These are distributed among 12 major phylogenetic clades $[23,28,29]$.

Accumulating circumstantial evidence suggests that Southeast and East Asia might be one center of origin of the genus. This included the common occurrence of both mating types of several heterothallic Phytophthora species, the occurrence of many Phytophthora diseases on mainly non-native horticultural trees and crops, and the apparent absence of Phytophthora diseases in natural ecosystems, despite the presence of species which cause severe forest dieback elsewhere $[2,10,12,13,15,16,30-35]$. In 2013, a survey in natural forests and streams of Taiwan demonstrated remarkably high diversity including ten described species and 17 previously unknown taxa of which nine were of hybrid origin. The results suggested that most of these taxa including the A1 mating type of P. cinnamomi were indigenous to Taiwan, whereas the A2 mating type of P. cinnamomi is introduced; that major Phytophthora phylogenetic clades 2, 5, 6, 7 and 9 are native to Southeast and Eastern Asia; and that interspecific hybridisation may have a major role in speciation and radiations in diverse natural ecosystems $[10,22]$.

The high Phytophthora diversity in Taiwan probably reflects both the high floristic, geological, and climatic diversity of this island and repeated immigration of Phytophthora species from mainland Asia via temporary landbridges during glacial periods in the pleistocene followed by periods of separation and speciation during interglacials [10,22,36-39]. Similarly, due to its complex geology, geomorphology, and orographic climates and the repeated immigration of plant species from both northern latitudes and the numerous islands of Sundaland during glacial periods, Indochina is also a biodiversity hotspot, harbouring $20 \%-25 \%$ of the world' s plant species [39-41]. With a north-south extension of $1650 \mathrm{~km}$ and a west-east extension ranging between 50 and $600 \mathrm{~km}$, Vietnam is located between $8^{\circ} 30^{\prime}$ and $23^{\circ} 30^{\prime}$ northern latitude and $102^{\circ} 10^{\prime}$ and $109^{\circ} 27^{\prime}$ eastern longitude in eastern Indochina along the South China Sea, covering approximately $330,000 \mathrm{~km}^{2}$. In Vietnam, seven climatic regions are distinguished. In simple terms, northern Vietnam has a humid subtropical monsoon climate with cool winters and hot rainy summers in lowland areas and cold misty winters and warm rainy summers in montane regions. Southern Vietnam has a tropical monsoon climate with warm winters and hot summers and a pronounced rainy period between May and October due to the East Asian monsoon. However, regionally, temperature and precipitation patterns can vary considerably due to orographic influences. The geology and geomorphology of Vietnam are also highly complex. Due to this environmental heterogeneity, the flora of Vietnam is remarkably diverse, comprising more than 10,350 species and 2256 genera of vascular plants, of which $10 \%$ and $3 \%$, respectively, are endemic [40]. This includes 245 and 211 native species of the Lauraceae and Fagaceae respectively, families known for the high susceptibility of their European and North American members to introduced Phytophthora species [5-7,42-44]. Therefore, as in Taiwan, a high diversity of unknown Phytophthora species might be expected in Vietnam. Further, due to their co-evolution with Vietnamese tree genera also present in Europe and North America, some of these might pose a threat to forests and natural ecosystems in the latter two continents. 
In spring 2016 and 2017, in the frame of a collaborative research project between the Mendel University in Brno, Forest Research and the University of Sassari, a survey of Phytophthora diversity was performed in a diverse range of natural forest types and river systems across Vietnam. This paper reports on the results of this Phytophthora survey and the association of Phytophthora spp. with disease symptoms of forest trees in Vietnam, and discusses the potential threat posed by previously unknown Phytophthora spp. to European and North American forests.

\section{Material and Methods}

\subsection{Sampling and Phytophthora Isolation}

Twenty-five natural forest stands covering a wide range of tree species, climates, and landscapes across Vietnam were selected for sampling (Figures 1 and 2). The forest stands were located in northern Vietnam in Hoàng Liên National Park (NP) (12 stands) and on two neighboring mountains (two stands), in Ba Vì NP (three stands) and in Cuc Phuong NP (five stands), and in southern Vietnam in Bù Gia Mập NP, U Minh Hạ NP and Côn Đảo NP on Côn Đảo island (each one stand). In addition, 16 rivers and streams were sampled in northern Vietnam (Figures 1 and 2c). Soil sampling and isolation methodology were according to $[4,10]$. In total, 91 rhizosphere soil samples were taken from 142 mature specimens of 64 native tree and shrub species. Three $20 \times 30 \times 20 \mathrm{~cm}$ soil monoliths were taken around each tree, at a distance of 30-150 cm from the stem base and at a soil depth of 10-30 cm. Aliquots of ca. 2 litres of rhizosphere soil together with roots (diameter $\leq 5 \mathrm{~mm}$ ) from all monoliths were bulked, and subsamples of ca. $200 \mathrm{~mL}$ were used for isolation tests. Isolations from soil samples were carried out at $18-20^{\circ} \mathrm{C}$ in an airconditioned laboratory at natural light using 3- to 10-day-old leaflets of native tree species, mainly Lithocarpus bacgangensis, L. corneus, Quercus glauca, Q. chapaensis, $Q$. gilva, Castanopsis indica and Chamaecyparis hodginsii, and the introduced Acacia mangium as baits floated over flooded soil. Brownish leaflets were examined at $\times 80$ under a light microscope for presence of Phytophthora sporangia. Infected leaflets were blotted dry, necrotic lesions cut into small segments and plated onto selective PARPNH agar (V8-juice agar (V8A) amended with $10 \mu \mathrm{g} / \mathrm{mL}$ pimaricin, 200 $\mu \mathrm{g} / \mathrm{mL}$ ampicillin, $10 \mu \mathrm{g} / \mathrm{mL}$ rifampicin, $25 \mu \mathrm{g} / \mathrm{mL}$ pentachloronitrobenzene (PCNB), $50 \mu \mathrm{g} / \mathrm{mL}$ nystatin and $50 \mu \mathrm{g} / \mathrm{mL}$ hymexazol).

In forest stand F07, the isolation of Phytophthora was also attempted from a bleeding bark lesion on a surface root of a mature Castanopsis acuminatissima (Figure 3e). Necrotic bark pieces were transported in distilled water to the lab and blotted dry on filter paper. Then, ca. $2 \mathrm{~mm}$ pieces were cut from the lesion margins and plated onto PARPNH agar.

In forest stand F11, freshly fallen leaves of a mature Rhododendron arboreum with necrotic lesions were collected from the forest floor close to forest stream R05 ca. $1 \mathrm{~m}$ above the waterline. The isolation of Phytophthora from these leaves was carried out as described below for leaves collected from rivers. 


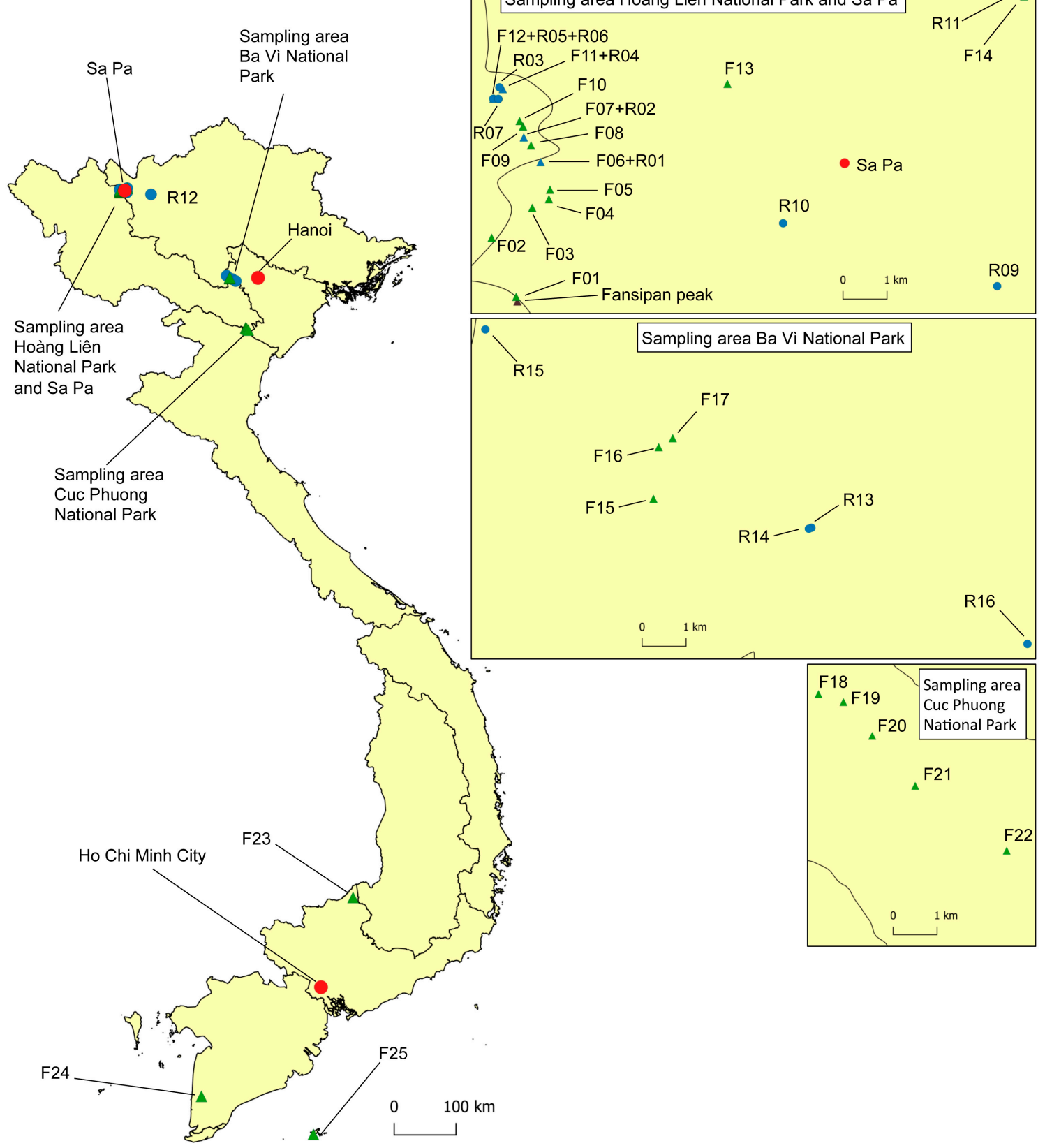

Figure 1. Location of the 25 forest sites (F01-F25; green triangles) and the 16 riparian sites (R01-R16; blue dots) included in the Phytophthora survey in Vietnam; blue triangles represent sites included in both the riparian and forest survey. For geographical coordinates and details of sites see Tables 1 and 2. 

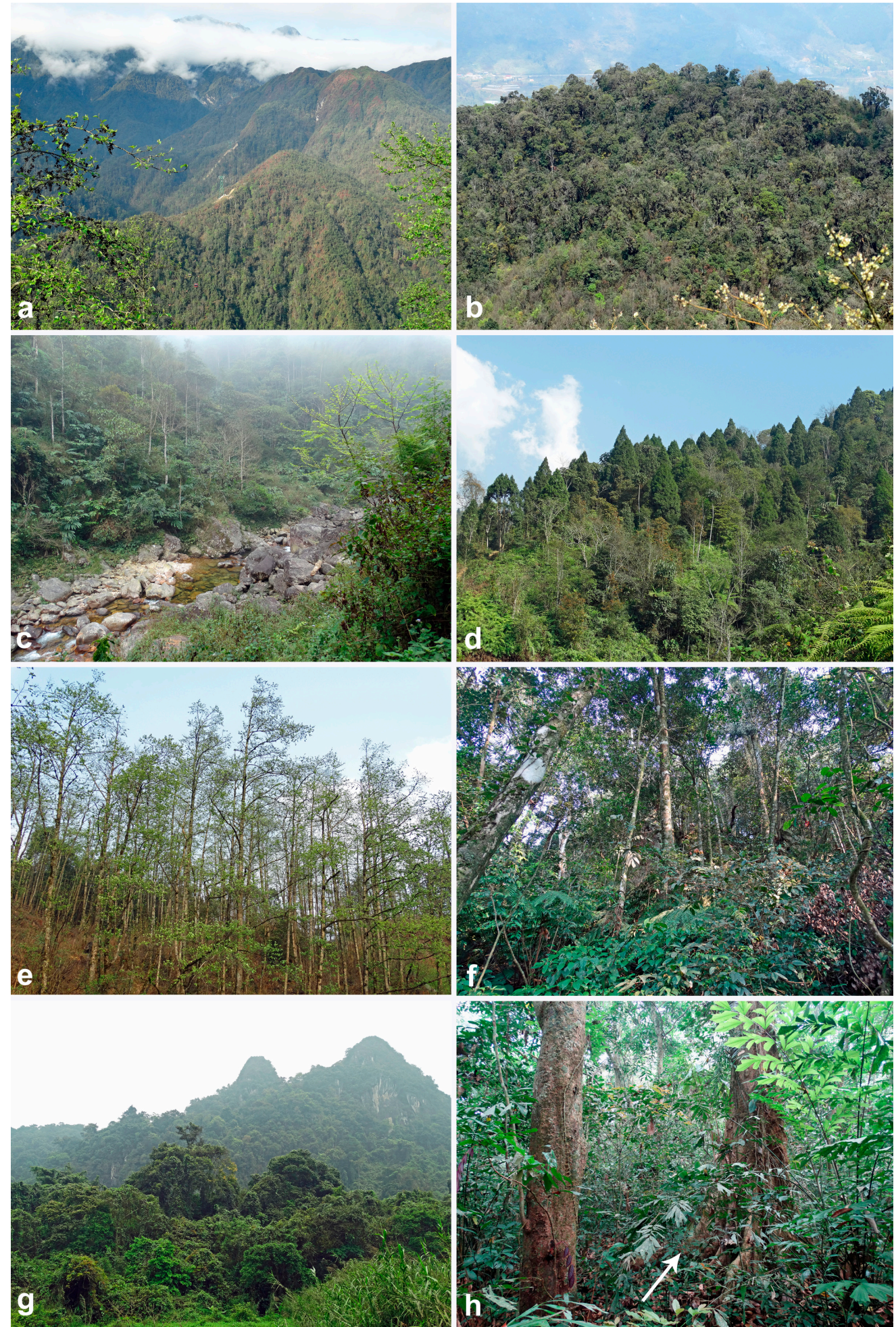

Figure 2. Representative forest stands and streams sampled in Vietnam; (a) Hoàng Liên National Park around the Fansipan mountain with diverse montane evergreen cloud forests and montane evergreen broadleaved forests; (b) diverse montane evergreen cloud forest F04 in Hoàng Liên National Park dominated by Fagaceae and Lauraceae species; (c) Cat Cat River (R10) running through a diverse montane evergreen forest in Hoàng Liên National Park; (d) montane Chamaecyparis hodginsii-Quercus forest on Sau Chua mountain; (e) montane Alnus nepalensis stand on Xin Chài mountain; (f) diverse, suptropical, humid evergreen forest F15 in Ba Vì National Park; (g) Cuc Phuong National Park with diverse, tropical, evergreen lowland rainforests growing on limestone; (h) diverse, tropical, evergreen lowland rainforest stand F20. For GPS coordinates see Tables 1 and 2; for location of sites see Figure 1. 

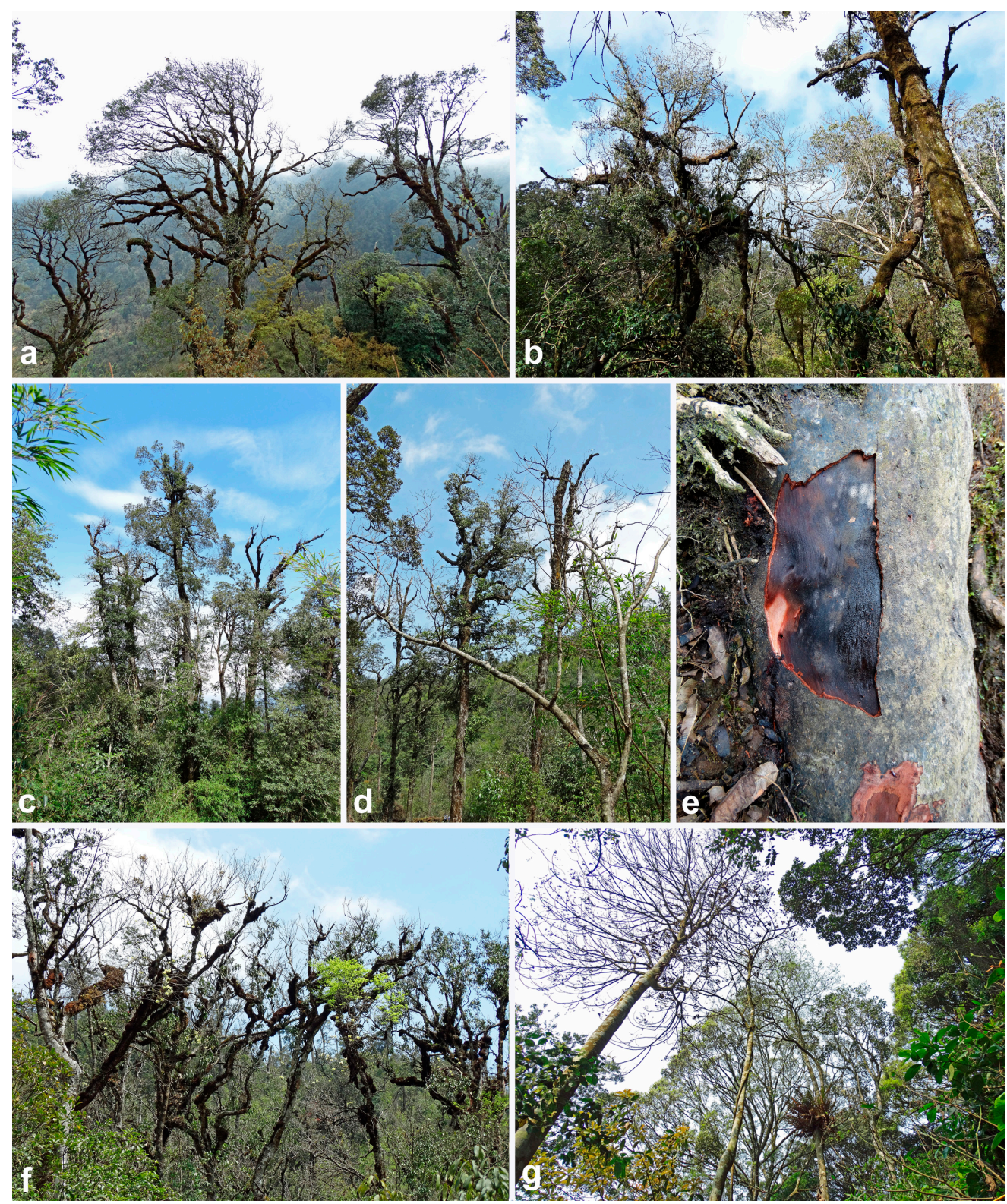

Figure 3. Disease symptoms of mature native trees in natural forest stands in Vietnam associated with presence of Phytophthora species in the rhizosphere; (a-f) montane evergreen cloud forests in Hoàng Liên National Park; (a) crown thinning and dieback of Quercus glauca in forest stand F03 (2337 m a.s.1.; P. cinnamomi A2); (b) crown dieback and mortality of Castanopsis acuminatissima and Neolitsea poilanei in forest stand F05 (2249 m a.s.l.; P. attenuata, P. castaneae, P. cinnamomi A2); (c,d,f) severe crown dieback and mortality of $C$. acuminatissima in a swampy depression of forest stand F06 close to stream R01 (2083 m a.s.l.; P. castaneae, P. cinnamomi A2, P. gregata); (f) the white flowers and young leaves in the crowns of $C$. acuminatissima belong to the epiphytic Rhododendron leptocladus; (e) bark lesion with staining of the underlying cambium caused by P. cinnamomi A2 on a surface root of C. acuminatissima in forest stand F07; (g) mortality of Dysoxylum juglans in suptropical humid evergreen forest stand F15 in Ba Vi National Park (1108 m a.s.l.; P. sp. attenuata-like 3).

For the isolation of Phytophthora spp. from the 16 rivers and streams, an in-situ baiting technique was used [10,11]. Twelve of the 16 riparian baiting sites were located inside or downstream of natural forests (Figure 2c). At each site, 15-20 non-wounded young leaves of the native C. indica, Citrus sinensis, L. bacgangensis, Q. glauca, and, in some cases, Carpinus sp., C. hodginsii, Cinnamomum iners, Dipterocarpus alatus, Prunus sp., Q. gilva and A. mangium were placed as baits in a $25 \times 30 \mathrm{~cm}$ raft, prepared using 
fly mesh and styrofoam, and the raft put to float at a place where water flow was calm. The rafts were collected after 2-3 days. In addition, in 2017 freshly fallen leaves of different tree species and flowers of Rhododendron arboreum and R. leptocladus were collected from forest streams R01, R02, R10 and R11. Baiting leaves and the collected fallen leaves and flowers were washed in distilled water and blotted dry on filter paper. Five to ten pieces (approximately $2 \times 2 \mathrm{~mm}$ ) were cut from the margins of each watersoaked or necrotic lesion of each leaf or flower, blotted on filter paper and plated onto PARPNH agar.

All Petri dishes with plated leaf, flower or bark pieces were incubated at $20^{\circ} \mathrm{C}$ in the dark and repeatedly examined under the stereo microscope at $\times 20$ for Phytophthora-like hyphae after $12-48 \mathrm{~h}$. Pure cultures were obtained by transferring single hyphal tips from the edge of the colonies onto V8A. Stock cultures were maintained on carrot agar (CA) [45] at $10{ }^{\circ} \mathrm{C}$ in the dark.

\subsection{Molecular Identification of Isolates}

For all Phytophthora isolates obtained in this study mycelial DNA was extracted from one-week old V8A cultures. Total DNA was extracted using the Phire Plant Direct PCR Kit (Thermo Fisher Scientific Inc., Waltham, MA USA) following the manufacturer's instructions. DNA was stored at $-20{ }^{\circ} \mathrm{C}$ until further use. For all isolates the region spanning the internal transcribed spacer (ITS1-5.8S-ITS2) region of the ribosomal DNA was amplified using primer-pairs ITS1/ITS4 or ITS6/ITS4 [29,46]. For representative isolates of several known and all putative new species the mitochondrial cox1 gene was amplified with both primer-pairs COXF4N/COXR4N and FM84/FM83 [47,48]. The PCR reaction mixture and the amplification conditions for ITS and cox 1 were according to $[29,47,48]$. PCR consumables were provided by Thermo Fisher Scientific. PCR products were purified and sequenced by GATC Biotech (Konstanz, Germany) and by Source Bioscience (Nottinham, UK) in both directions with the primers used for PCR amplification.

Sequences were edited using Geneious (Version 11.1.2, Biomatters Ltd., Auckland, New Zealand). Heterozygous sites observed were labelled according to the IUPAC coding system. Consensus sequences were aligned using the CLUSTAL W algorithm. The consensus sequences were subjected to an NCBI BLAST search (http://www.ncbi.nlm.nih.gov/BLAST/) and to a blast search in a local database containing sequences of ex-type isolates or key isolates from published studies to identify the closest related sequences. Isolates were assigned to a species when sequence identities were above a 99\% cut-off in respect to those of ex-type isolates or key isolates. ITS and cox1 sequences from representative isolates of all known and all putative new Phytophthora species obtained in this study were deposited at GenBank and accession numbers are given in Supplementary Table S1.

\subsection{Classical Identification of Isolates}

Colony growth patterns of 7-d-old cultures grown at $20^{\circ} \mathrm{C}$ in the dark on V8A, malt-extract agar (MEA; Oxoid Ltd., Basingstoke, UK) and PDA [21] and morphological characters of sporangia, oogonia, antheridia, chlamydospores, hyphal swellings, and aggregations were compared with isolates from known species and with species descriptions in the literature.

Sporangia production and microscopic examinations and measurements of morphological structures at $\times 400$ were according to $[21,22]$ using a compound microscope (Zeiss Axioimager.Z2, Carl Zeiss AG, Oberkochen, Germany), a digital camera (Zeiss Axiocam ICc5) and a biometric software (Zeiss ZEN). Self-sterile isolates were paired on both V8A and CA with known A1 and A2 mating type tester strains of P. cinnamomi, P. $\times$ cambivora and $P$. $\times$ heterohybrida (isolates with non-papillate sporangia) or P. botryosa, P. colocasiae and P. meadii (isolates with papillate sporangia). All pairings were examined after 4-6 weeks incubation at $20^{\circ} \mathrm{C}$ in the dark in order to determine whether self-sterile isolates are heterothallic or sterile and to which mating type heterothallic isolates belong [21]. All isolates are preserved in the culture collections maintained at Mendel University and Forest Research. 


\section{Results}

In total, 943 oomycete isolates, including 652 Phytophthora isolates and 291 isolates from other oomycete genera, were obtained from forest stands (Table 1) and river systems (Table 2) in Vietnam. The Phytophthora isolates belonged to 13 described species, five informally designated taxa and 21 previously unknown taxa. From the other oomycete genera, 122 isolates were identified to species level. They could be assigned to the recently described Nothophytophthora vietnamensis (26 isolates), Phytopythium vexans sensu lato (63 isolates from 14 partly highly different haplotypes), four other known species and three novel taxa of Phytopythium (16 isolates), two described species and six novel taxa of Pythium (17 isolates) and one novel taxon of Elongisporangium. The remaining 169 isolates, which were not identified to species level, belonged to Phytopythium (161 isolates), Pythium (7 isolates) and Saprolegnia (1 isolate), respectively. GenBank accession numbers of ITS sequences of representative isolates of all oomycete taxa and of $\operatorname{cox} 1$ sequences of representative isolates of most Phytophthora taxa are given in Supplementary Table S1. Detailed descriptions of morphological characteristics, morphometric and temperature-growth data, and multigene phylogenies for all new Phytophthora species will be presented in separate publications.

\subsection{Phytophthora Diversity in Natural and Semi-Natural Forest Stands}

In 20 forest stands (80\%), 20 Phytophthora taxa were isolated from 58 of the 91 soil samples $(63.7 \%)$ taken from the rhizosphere of 52 of the 64 woody plant species sampled $(81.3 \%)$; from the root lesion of C. acuminatissima in stand F07; and from all four freshly fallen Rhododendron leaves collected from the ground in stand F11: P. attenuata, P. castaneae, P. chlamydospora, P. cinnamomi, P. gregata, P. heveae, P. parvispora, P. ramorum, P. citricola VII, three new species related to $P$. attenuata, three new species from the 'Phytophthora citricola complex', three new species related to P. botryosa and P. meadii, and one new species related to P. multivesiculata and another to P. tropicalis, respectively (Table 1). From 29 of the 35 Phytophthora-negative soil samples, several known and previously unknown Phytopythium or Pythium spp. were isolated (Table 1). The only forest site from which no oomycete isolates could be obtained was subalpine Rhododendron scrub at 2903 m altitude near the Fansipan peak (F01).

Phytophthora cinnamomi, Clade 7c, was isolated from 26 of 66 rhizosphere soil samples (39.4\%) collected from 27 of the 50 tree and shrub species (54\%) in 13 of the 17 mountainous forest stands sampled $(76.5 \%)$, making it the most widespread and common Phytophthora species above $700 \mathrm{~m}$ altitude. The A2 mating type of P. cinnamomi was present in 11 forest stands with an altitudinal amplitude ranging from 713 to $2337 \mathrm{~m}$ above sea level (a.s.1.). In contrast, the A1 mating type was only found in four forest stands located between 1108 and $2636 \mathrm{~m}$ a.s.l. (Figure 1; Table 1). Both mating types co-occurred in one stand in Hoàng Liên NP and another in Ba Vì NP. Interestingly, in Hoàng Liên NP, the A1 mating type was present in the upper montane Rhododendron forest F02 at $2636 \mathrm{~m}$ a.s.l. and in the lower montane stands F11 and F12 at $1900 \mathrm{~m}$ a.s.l., but was not detected in the eight forest stands (F03-F10) sampled between 2337 and $2022 \mathrm{~m}$ a.s.1., all highly infested by the A2 mating type. The latter was also isolated from a bark lesion on a surface root of C. acuminatissima in stand F07. Two A2 isolates from stand F11 were able to produce oogonia in single culture on V8A (Table 1). Over all stands, the A1:A2 mating type ratio of the $151 \mathrm{P}$. cinnamomi isolates was 30.5:69.5, whereas in the two stands with co-occurrence of both mating types the A1:A2 ratio of the 44 isolates was 59.1:40.9. Among the 39 P. cinnamomi isolates for which ITS sequences were produced, 32 isolates belonged to the same haplotype as the ex-type isolate from Sumatra (CBS 142.22; GenBank accession no. KU899160) (Table S1). Six isolates, representing both mating types, from stands F02 and F05 in Hoàng Liên NP and stand F17 in Ba Vì NP were heterozygous at position 767 (K instead of G) (Table 1S) and shared the same haplotype with an isolate from a subtropical Quercus forest in Taiwan (TW213; GenBank accession no. KU682570). Another isolate from stand F17 shared the heterozygous position 767 and was also heterozygous at position 89 (Y instead of C) (Supplementary Table S1). The 15 isolates for which the cox 1 gene was sequenced belonged to eight different haplotype which differed over a $712 \mathrm{bp}$ alignment from the ex-type isolate (KU899315) at 1-4 positions. 
Phytophthora parvispora was exclusively found in stand F15 in Ba Vì NP where it co-occurred with both mating types of its closest relative P. cinnamomi (Table 1). Compared to the ex-type of P. parvispora (CBS 132772; KC478667), the three isolates had identical cox1 sequences and differed in ITS by one heterozygous site at position 73 (Y instead of T) (Supplementary Table S1). In mating tests with A1 and A2 tester strains of P. cinnamomi, all isolates were sterile.

Phytophthora attenuata from Clade 7a and three previously unknown taxa closely related to P. attenuata were recovered from five forest stands in Hoàng Liên NP and Ba Vì NP (Table 1). The individual taxa from this ' $P$. attenuata complex' differed in their altitudinal amplitude and geographical distribution (Figure 1; Table 1). Phytophthora attenuata, P. sp. attenuata-like 1 and $P$. sp. attenuata-like 2 were only found in Hoàng Liên NP. Most widespread was $P$. sp. attenuata-like 1 which was isolated from the rhizosphere of five tree species in three stands located between 2249 and $2636 \mathrm{~m}$ a.s.l., followed by P. attenuata (three tree species in two stands; 1910-2249 $\mathrm{m}$ a.s.1.) and $P$. sp. attenuata-like 2 (2 tree species in 1 stand; $1910 \mathrm{~m}$ a.s.l.). In contrast, $P$. sp. attenuata-like 3 was exclusively found between 713 and $1108 \mathrm{~m}$ altitude in two of the three forest stands sampled in Ba Vi NP where it was associated with six tree species (Table 1). The ITS and cox 1 sequences of P. attenuata isolates from Vietnam differed from the ex-type isolate (CBS 141199; GenBank nos. KU517154 and KU517148) and other isolates of P. attenuata from Taiwan at 0-1 and 0-5 positions. Phytophthora sp. attenuata-like $1, P$. sp. attenuata-like 2 and $P$. sp. attenuata-like 3 showed differences to $P$. attenuata in ITS at $0-1,1-2$ and 2-3 positions, respectively, and in cox1 at 6-8, 9-11 and 6-8 positions, respectively. The cox 1 sequences of the three new taxa differed from each other at 8-17 positions. Heterozygous sites were present in the ITS sequences of all isolates of $P$. sp. attenuata-like 2 (R at position 184) and most isolates of $P$. sp. attenuata-like 3 ( $\mathrm{Y}$ in position 54; $\mathrm{K}$ in position 152). The ITS sequence of one isolate of $P$. sp. attenuata-like 1 from stand F05 contained seven heterozygous sites possibly suggesting hybrid origin.

Phytophthora castaneae from Clade 5 showed a similar altitudinal (1108-2242 $\mathrm{m}$ a.s.1.) and geographical distribution to P. cinnamomi (Figure 1; Table 1). It was isolated from the rhizosphere of 13 tree species from the genera Castanopsis, Lithocarpus, Neolitsea, Meliosma, Illicium and Rhododendron in seven stands in Hoàng Liên NP and Ba Vì NP, and C. hodginsii in stand F14 on Sau Chua mountain where it was the only Phytophthora species recovered (Table 1). The ITS sequences of all isolates from Hoàng Liên NP and several isolates from Ba Vì NP matched the ex-type of P. castaneae (ICMP 19434; GenBank no. KP295319). However, several isolates from Ba Vi NP had a unique polymorphism at position 54 (A or $R$ instead of $G$ ) while all isolates from Sau Chua mountain were characterised by having a unique polymorphism at position 590 (A instead of $\mathrm{G}$ ). The cox1 sequences of 15 isolates from the seven stands constituted six haplotypes which differed from the ex-type isolate (KP295234) by 0-1 bp. Interestingly, all four tested isolates from Sau Chua mountain had a unique polymorphism at position 421 (A instead of G). Five of the six tested $P$. castaneae isolates from stand F15 in Ba Vì NP shared a $\mathrm{T}$ at position 369 with $P$. heveae isolates from the same stand and with the P. heveae ex-type (CBS296.29; GenBank nos. HQ643238 and KP295326) whereas P. castaneae isolates from the other stands and the $P$. castaneae ex-type have a $C$ at this position. Compared to $P$. castaneae, the other Clade 5 species found in this survey, P. heveae, had a lower altitudinal amplitude. Phytophthora heveae was isolated from the rhizosphere of 10 tree species in the subtropical lower montane stands F15 and F16 in Ba Vì NP and in four tropical lowland rainforest stands in Cuc Phuong NP, Bù Gia Mập NP and Côn Đảo NP (Figure 1; Table 1). Both Clade 5 species only co-occurred in stand F15. The ITS sequences of all $P$. heveae isolates (Table S1) matched the ex-type of $P$. heveae. The cox 1 sequences of all isolates differed from the ex-type (GenBank no. KP295239) at position 536 (T instead of C). Isolates from Cuc Phuong NP and Bù Gia Mập NP had unique polymorphisms at positions 30 (C instead of A) and 390 (A instead of T), respectively. The morphology of all isolates of $P$. castaneae and P. heveae was in accordance with the original descriptions [2]. 
Table 1. Location, altitude, geological substrate and vegetation of 25 forest sites sampled in spring 2016 and 2017 in Vietnam, sampled tree species and Phytophthora and other oomycete taxa isolated.

\begin{tabular}{|c|c|c|c|c|c|c|c|}
\hline Site no. & $\begin{array}{c}\text { GPS } \\
\text { Coordinates }\end{array}$ & $\begin{array}{l}\text { Altitude } \\
\text { (m a.s.l) }\end{array}$ & Location & $\begin{array}{l}\text { Geological } \\
\text { Substrate }\end{array}$ & Vegetation & $\begin{array}{c}\text { Sampled Tree Species } \\
\text { (no. of Phytophthora-Positive/ Sampled Trees) }\end{array}$ & $\begin{array}{c}\text { Phytophthora and } \\
\text { Nothophytophthora spp. } \\
\text { (no. of Positive Samples) } \mathrm{a}, \mathrm{b}\end{array}$ \\
\hline F01 & $\begin{array}{l}\text { N22 } 18.466 \\
\text { E103 } 46.480\end{array}$ & 2903 & $\begin{array}{l}\text { Fansipan, Hoàng Liên } \\
\text { National Park (NP) }\end{array}$ & $\begin{array}{l}\text { Triassic schists and } \\
\text { sandstones }\end{array}$ & $\begin{array}{c}\text { Subalpine } \\
\text { Rhododendron scrub }\end{array}$ & Rhododendron spp. (0/3) & - \\
\hline F02 & $\begin{array}{l}\text { N22 } 19.194 \\
\text { E103 } 46.177\end{array}$ & 2636 & $\begin{array}{l}\text { Fansipan, Hoàng Liên } \\
\text { NP }\end{array}$ & $\begin{array}{l}\text { Triassic schists and } \\
\text { sandstones }\end{array}$ & $\begin{array}{l}\text { Upper montane } \\
\text { Rhododendron } \\
\text { ('Elfin') cloud forest }\end{array}$ & $\begin{array}{l}\text { Rhododendron arboreum, mix from } 3 \text { trees with } \\
\text { dieback (DB) (1/1) }\end{array}$ & ATT1 (1), CIN A1 (1) \\
\hline F03 & $\begin{array}{l}\text { N22 } 19.563 \\
\text { E103 } 46.679\end{array}$ & 2337 & Hoàng Liên NP & $\begin{array}{l}\text { Triassic schists and } \\
\text { sandstones }\end{array}$ & $\begin{array}{l}\text { Montane evergreen } \\
\text { cloud forest }\end{array}$ & Quercus glauca, DB (2/2) & CIN A2 (2) \\
\hline F04 & $\begin{array}{l}\text { N22 } 19.670 \\
\text { E103 } 46.885\end{array}$ & 2242 & Hoàng Liên NP & $\begin{array}{l}\text { Triassic schists and } \\
\text { sandstones }\end{array}$ & $\begin{array}{l}\text { Montane evergreen } \\
\text { cloud forest }\end{array}$ & $\begin{array}{l}\text { Meliosma henryi }(1 / 1) \\
\text { Betula alnoides \& Elaeocarpus japonicus }(1 / 1) \\
\text { Castanopsis acuminatissima, mix from } 2 \text { trees, DB } \\
\text { (1/1) } \\
\text { C. acuminatissima with DB \& Acer campbellii (1/1) }\end{array}$ & $\begin{array}{c}\text { CAS (1) } \\
\text { ATT1 (1), CIN A2 (1) } \\
\text { ATT1 (1), CIN A2 (1) } \\
\text { VIE (1) }\end{array}$ \\
\hline F05 & $\begin{array}{l}\text { N22 } 19.786 \\
\text { E103 } 46.899\end{array}$ & 2249 & Hoàng Liên NP & $\begin{array}{l}\text { Triassic schists and } \\
\text { sandstones }\end{array}$ & $\begin{array}{l}\text { Montane evergreen } \\
\text { cloud forest }\end{array}$ & $\begin{array}{l}\text { Neolitsea poilanei, } \mathrm{DB}(3 / 3) \\
\text { C. acuminatissima mix from } 3 \text { trees, DB }(1 / 1) \\
\text { Illicium griffithii \& C. acuminatissima, DB (1/1) }\end{array}$ & $\begin{array}{c}\text { ATT1 (3), CAS (1), CIN A2 (3) } \\
\text { ATT (1), CIN A2 (1) } \\
\text { CAS (1) }\end{array}$ \\
\hline F06 & $\begin{array}{l}\text { N22 } 20.127 \\
\text { E103 } 46.782\end{array}$ & 2083 & Hoàng Liên NP & $\begin{array}{l}\text { Triassic schists and } \\
\text { sandstones }\end{array}$ & $\begin{array}{l}\text { Montane evergreen } \\
\text { cloud forest }\end{array}$ & $\begin{array}{l}\text { C. acuminatissima, DB }(2 / 2) \\
\text { M. henryi \& A. campbellii }(1 / 1) \\
\text { M. henryi \& Neolitsea merilliana }(1 / 1)\end{array}$ & $\begin{array}{c}\text { CIN A2 (2), GRE (1), CAS (1) }{ }^{\mathrm{d}} \\
\text { GRE (1) } \\
\text { CIN A2 (1), MUV1 (1) }\end{array}$ \\
\hline F07 & $\begin{array}{l}\text { N22 } 20.430 \\
\text { E103 } 46.574\end{array}$ & 2010 & Hoàng Liên NP & $\begin{array}{l}\text { Triassic schists and } \\
\text { sandstones }\end{array}$ & $\begin{array}{l}\text { Montane evergreen } \\
\text { cloud forest }\end{array}$ & $\begin{array}{l}\text { Illicium tsaii } \mathcal{E} \text { Rhododendron sinofalconeri }(1 / 1) \\
\text { C. acuminatissima, DB, necrotic root lesion }(1 / 1)\end{array}$ & $\begin{array}{l}\text { CAS (1) } \\
\text { CIN A2 (1) }\end{array}$ \\
\hline F08 & $\begin{array}{l}\text { N22 } 20.331 \\
\text { E103 } 46.664\end{array}$ & 2066 & Hoàng Liên NP & $\begin{array}{l}\text { Triassic schists and } \\
\text { sandstones }\end{array}$ & $\begin{array}{l}\text { Montane evergreen } \\
\text { cloud forest }\end{array}$ & $\begin{array}{l}\text { Casearia annamensis }(1 / 1) \\
\text { Acer oblongum, mix from } 2 \text { trees }(0 / 1)\end{array}$ & $\underset{-\mathrm{e}}{\mathrm{C}}$ \\
\hline F09 & $\begin{array}{l}\text { N22 } 20.565 \\
\text { E103 } 46.565\end{array}$ & 2010 & Hoàng Liên NP & $\begin{array}{l}\text { Triassic schists and } \\
\text { sandstones }\end{array}$ & $\begin{array}{c}\text { Montane evergreen } \\
\text { cloud forest }\end{array}$ & $\begin{array}{l}\text { C. acuminatissima }(0 / 1) \\
\text { Q. glauca }(0 / 2)\end{array}$ & $\begin{array}{c}-\mathrm{e} \\
- \\
\end{array}$ \\
\hline F10 & $\begin{array}{l}\text { N22 } 20.632 \\
\text { E103 } 46.523\end{array}$ & 2022 & Hoàng Liên NP & $\begin{array}{l}\text { Triassic schists and } \\
\text { sandstones }\end{array}$ & $\begin{array}{l}\text { Montane, evergreen } \\
\text { cloud forest }\end{array}$ & $\begin{array}{l}\text { Neolitsea polycarpa, mix from } 3 \text { trees, DB }(1 / 1) \\
\text { N. polycarpa, Symplocos pseudobarberina \& } \\
\text { Beilschmiedia roxburghiana }(1 / 1)\end{array}$ & $\begin{array}{l}\text { CIN A2 (1) } \\
\text { CIN A2 (1) }\end{array}$ \\
\hline F11 & $\begin{array}{l}\text { N22 } 21.026 \\
\text { E103 } 46.315\end{array}$ & 1910 & Hoàng Liên NP & $\begin{array}{l}\text { Triassic schists and } \\
\text { sandstones }\end{array}$ & $\begin{array}{c}\text { Montane, evergreen } \\
\text { broadleaved forest }\end{array}$ & $\begin{array}{l}\text { A. oblongum \& Symplocos dryophila }(2 / 2) \\
\text { C. acuminatissima DB, Ilex leseeneri \& Eurya } \\
\text { annamensis }(1 / 1) \\
\text { R. arboreum }(1 / 1)^{g}\end{array}$ & $\begin{array}{l}\text { ATT (1), ATT2 (1), CIN A1 (2), } \\
\text { CIN A2 (1), CIN A2ho (1) e,f } \\
\text { CIN A2 (1), CAS (1) } \\
\text { CHL (1), RAM A1 (1) }\end{array}$ \\
\hline
\end{tabular}


Table 1. Cont

\begin{tabular}{|c|c|c|c|c|c|c|c|}
\hline Site no. & $\begin{array}{c}\text { GPS } \\
\text { Coordinates }\end{array}$ & $\begin{array}{l}\text { Altitude } \\
\text { (m a.s.l) }\end{array}$ & Location & $\begin{array}{l}\text { Geological } \\
\text { Substrate }\end{array}$ & Vegetation & $\begin{array}{c}\text { Sampled Tree Species } \\
\text { (no. of Phytophthora-Positive/ Sampled Trees) }\end{array}$ & $\begin{array}{c}\text { Phytophthora and } \\
\text { Nothophytophthora spp. } \\
\text { (no. of Positive Samples) }{ }^{\mathrm{a}, \mathrm{b}}\end{array}$ \\
\hline F12 & $\begin{array}{l}\text { N22 } 20.909 \\
\text { E103 } 46.199\end{array}$ & 1895 & Hoàng Liên NP & $\begin{array}{l}\text { Triassic schists and } \\
\text { sandstones }\end{array}$ & $\begin{array}{c}\text { Montane, evergreen } \\
\text { broadleaved forest }\end{array}$ & $\begin{array}{l}\text { Acer oliverianum, Eryobotrya cavaleriei \& } \\
\text { Symplocos quillaminii }(1 / 1) \\
\text { Q. glauca }(1 / 1)\end{array}$ & $\begin{array}{l}\text { CIN A1 (1) } \\
\text { CIN A1 (1) }\end{array}$ \\
\hline F13 & $\begin{array}{l}\text { N22 } 21.090 \\
\text { E103 } 49.092\end{array}$ & 1717 & Xin Chài mountain & $\begin{array}{l}\text { Triassic schists and } \\
\text { sandstones }\end{array}$ & $\begin{array}{l}\text { Montane Alnus } \\
\text { forest on steep } \\
\text { loamy slope }\end{array}$ & Alnus nepalensis (2/3) & $\begin{array}{c}\operatorname{CIT} \text { VII (1), MEA1 (1), } \times \operatorname{TRO} 2(1), \\
\operatorname{VIE}^{\mathrm{e}}\end{array}$ \\
\hline F14 & $\begin{array}{l}\text { N22 } 22.168 \\
\text { E103 } 52.758\end{array}$ & 1367 & Sau Chua mountain & $\begin{array}{l}\text { Triassic schists and } \\
\text { sandstones }\end{array}$ & $\begin{array}{c}\text { Montane } \\
\text { Chamaecyparis-Quercus } \\
\text { forest }\end{array}$ & Chamaecyparis hodginsii (7/9) & CAS $(7)^{c, e}$ \\
\hline F15 & $\begin{array}{l}\text { N21 } 3.699 \\
\text { E105 } 21.733\end{array}$ & 1108 & $\begin{array}{l}\text { Ba Vì National Park } \\
\text { (NP) }\end{array}$ & $\begin{array}{l}\text { Triassic schists and } \\
\text { sandstones and } \\
\text { porphyrites }\end{array}$ & $\begin{array}{l}\text { Suptropical humid } \\
\text { evergreen forest }\end{array}$ & $\begin{array}{l}\text { Castanopsis chinensis (2/2) } \\
\text { C. chinensis \& Beilschmiedia fordii (1/1) } \\
\text { Dysoxylum juglans, DB }(1 / 1) \\
\text { Eberhardtia tonkinensis, Antidesma sp. \& Jasminum } \\
\text { sp. }(0 / 1) \\
\text { Eurya japonica \& Nephelium lappaceum (1/1) } \\
\text { Lithocarpus bacgangensis }(1 / 1) \\
\text { Lithocarpus pseudosundaicus }(0 / 1) \\
\text { Machilus bonii }(1 / 1) \\
\text { Magnolia annamensis }(1 / 1) \\
\text { Q. glauca, mix from } 3 \text { trees }(1 / 1) \\
\text { Vernicia montana \& Antidesma sp. (1/1) }\end{array}$ & $\begin{array}{c}\text { ATT3 (1), CAS (1), CIN A1 (1) }{ }^{\mathrm{e}} \\
\text { CAS (1), HEV (1), PAR (1) }{ }^{\mathrm{d}} \\
\text { ATT3 (1) } \\
-\mathrm{d}^{\mathrm{d}} \\
\text { CAS (1) }{ }^{\mathrm{e}} \\
\text { CAS (1) } \\
- \\
\text { CIN A1 (1) } \\
\text { CIN A2 (1) } \\
\text { e } \\
\text { ATT3 (1), CIN A1 (1), HEV (1), } \\
\text { PAR (1) }{ }^{\mathrm{e}} \\
\text { ATT3 (1), CIN A1 (1) } \mathrm{d}\end{array}$ \\
\hline F16 & $\begin{array}{l}\text { N21 } 04.455 \\
\text { E105 } 21.810\end{array}$ & 807 & Ba Vì NP & $\begin{array}{l}\text { Triassic schists and } \\
\text { sandstones and } \\
\text { porphyrites }\end{array}$ & $\begin{array}{l}\text { Suptropical humid } \\
\text { evergreen forest }\end{array}$ & $\begin{array}{l}\text { Caryodaphnosis baviensis }(0 / 2) \\
\text { Lithocarpus bacgangensis }(1 / 1) \\
\text { Meliosma arnottiana }(1 / 1) \\
\text { Phoebe petelotii, Machilus thunbergii \& Claoxylon } \\
\text { indicum }(1 / 1)\end{array}$ & $\begin{array}{l}-{ }^{\mathrm{e}} \\
\operatorname{HEV}(1)^{\mathrm{e}} \\
\operatorname{CIT~IX~(1)~}^{\mathrm{e}} \\
\operatorname{CIN~A2~(1)~}^{\mathrm{e}}\end{array}$ \\
\hline F17 & $\begin{array}{l}\text { N21 } 04.587 \\
\text { E105 } 22.016\end{array}$ & 713 & Ba Vì NP & $\begin{array}{l}\text { Triassic schists and } \\
\text { sandstones and } \\
\text { porphyrites }\end{array}$ & $\begin{array}{l}\text { Suptropical humid } \\
\text { evergreen forest }\end{array}$ & $\begin{array}{l}\text { Alsodaphne velutina \& Litsea brevipetiolata }(1 / 1) \\
\text { Bischofia javanica \& Litsea monocephala }(0 / 1) \\
\text { C. chinensis }(1 / 1) \\
\text { Castanopsis tonkinensis }(1 / 1) \\
\text { Q. glauca }(0 / 1)\end{array}$ & $\begin{array}{c}-\mathrm{e} \\
-\mathrm{e} \\
\operatorname{ATT} 3(1), \mathrm{CIN} \mathrm{A2} \mathrm{(1)} \\
\text { ATT3 (1) } \\
-\mathrm{e}^{\mathrm{e}}\end{array}$ \\
\hline
\end{tabular}


Table 1. Cont.

\begin{tabular}{|c|c|c|c|c|c|c|c|}
\hline Site no. & $\begin{array}{c}\text { GPS } \\
\text { Coordinates }\end{array}$ & $\begin{array}{l}\text { Altitude } \\
\text { (m a.s.l) }\end{array}$ & Location & $\begin{array}{l}\text { Geological } \\
\text { Substrate }\end{array}$ & Vegetation & $\begin{array}{l}\text { Sampled Tree Species } \\
\text { (no. of Phytophthora-Positive/ Sampled Trees) }\end{array}$ & $\begin{array}{c}\text { Phytophthora and } \\
\text { Nothophytophthora spp. } \\
\text { (no. of Positive Samples) a,b }\end{array}$ \\
\hline F18 & $\begin{array}{l}\text { N20 } 20.876 \\
\text { E105 } 35.793\end{array}$ & 392 & $\begin{array}{l}\text { Cuc Phuong National } \\
\text { Park (NP) }\end{array}$ & Triassic limestones & $\begin{array}{l}\text { Tropical evergreen } \\
\text { lowland rainforest }\end{array}$ & $\begin{array}{l}\text { C. baviensis } \mathcal{E} \text { Litsea robusta }(0 / 1) \\
\text { Dracontomelum duppereanum, mix from } 2 \text { trees } \\
(0 / 1) \\
\text { Saraca dives, mix from } 2 \text { trees }(1 / 1)\end{array}$ & $\begin{array}{c}-\mathrm{e} \\
-\mathrm{e} \\
\operatorname{HEV}(1)^{\mathrm{e}}\end{array}$ \\
\hline F19 & $\begin{array}{l}\text { N20 } 20.779 \\
\text { E105 } 36.099\end{array}$ & 356 & Cuc Phuong NP & Triassic limestones & $\begin{array}{l}\text { Tropical evergreen } \\
\text { lowland rainforest }\end{array}$ & $\begin{array}{l}\text { Allophylus cobbe, mix from } 2 \text { trees }(0 / 1) \\
\text { D. duppereanum \& S. dives }(0 / 2)\end{array}$ & $\begin{array}{c}-\mathrm{e} \\
-\mathrm{e}, \mathrm{h}\end{array}$ \\
\hline F20 & $\begin{array}{l}\text { N20 } 20.366 \\
\text { E105 } 36.452\end{array}$ & 318 & Cuc Phuong NP & Triassic limestones & $\begin{array}{l}\text { Tropical evergreen } \\
\text { lowland rainforest }\end{array}$ & $\begin{array}{l}\text { A. cobbe, Ficus sp., Merremia boisiana \& Homalium } \\
\text { sp. }(1 / 1) \\
\text { S. dives }(0 / 2)\end{array}$ & $\begin{array}{c}\text { MEA2 (1) } \\
-\mathrm{e}, \mathrm{i}\end{array}$ \\
\hline F21 & $\begin{array}{l}\text { N20 } 19.755 \\
\text { E105 } 36.979\end{array}$ & 267 & Cuc Phuong NP & Triassic limestones & $\begin{array}{l}\text { Tropical evergreen } \\
\text { lowland rainforest }\end{array}$ & $\begin{array}{l}\text { Anogeissus acuminata }(0 / 1) \\
\text { A. acuminata \& Taxotrophis macrophylla }(1 / 2)\end{array}$ & $\begin{array}{c}-\mathrm{d} \text { or e } \\
\operatorname{CIT} X(1)^{e, j}\end{array}$ \\
\hline F22 & $\begin{array}{l}\text { N20 } 18.963 \\
\text { E105 } 38.101\end{array}$ & 264 & Cuc Phuong NP & Triassic limestones & $\begin{array}{l}\text { Tropical evergreen } \\
\text { lowland rainforest }\end{array}$ & $\begin{array}{l}\text { C. baviensis }(0 / 1) \\
\text { C. baviensis \& } S \text {. dives }(0 / 1) \\
\text { S. dives, mix from } 2 \text { trees }(0 / 1)\end{array}$ & $\begin{array}{l}-e \\
-e, j \\
-e\end{array}$ \\
\hline F23 & $\begin{array}{l}\text { N12 } 06.326 \\
\text { E107 } 09.396\end{array}$ & 417 & $\begin{array}{l}\text { Bù Gia Mập National } \\
\text { Park }\end{array}$ & $\begin{array}{c}\text { Quaternary } \\
\text { alluvial sediments }\end{array}$ & $\begin{array}{l}\text { Tropical evergreen } \\
\text { lowland rainforest }\end{array}$ & $\begin{array}{l}\text { Dipterocarpus alatus, Ailanthus triphysa, Hopea } \\
\text { odorata \& Dalbergia oliveri (1/1) }\end{array}$ & $\operatorname{HEV}(1)^{\mathrm{e}}$ \\
\hline $\mathrm{F} 24$ & $\begin{array}{l}\text { N9 } 13.645 \\
\text { E104 } 57.330\end{array}$ & 4 & $\begin{array}{l}\text { U Minh Hạ National } \\
\text { Park }\end{array}$ & Quaternary peat & $\begin{array}{l}\text { Tropical lowland } \\
\text { peat forest }\end{array}$ & Melaleuca cajuputi (0/3) & $-k$ \\
\hline F25 & $\begin{array}{l}\text { N8 } 40.621 \\
\text { E106 } 34.836\end{array}$ & 55 & $\begin{array}{l}\text { Côn Đảo National } \\
\text { Park, Côn Lôn island }\end{array}$ & $\begin{array}{l}\text { Rhyolite and } \\
\text { diorite }\end{array}$ & $\begin{array}{l}\text { Tropical evergreen } \\
\text { lowland rainforest }\end{array}$ & $\begin{array}{l}\text { Chukrasia tabularis }(0 / 1) \\
\text { A. triphysa, } \text { C. tabularis }(1 / 1) \\
\text { Leucaena leucocephala, Canarium album \& Hopea } \\
\text { odorata }(1 / 1) \\
\text { H. odorata, C. album, D. alatus }(1 / 1)\end{array}$ & $\begin{array}{l}-\mathrm{e}, \mathrm{k} \\
\text { CIT XI }^{\mathrm{e}, \mathrm{l}} \\
\text { BOT2 }^{\mathrm{e}, \mathrm{j}} \\
\mathrm{HEV}^{\mathrm{l}, \mathrm{m}}\end{array}$ \\
\hline
\end{tabular}

${ }^{\mathrm{a}} \mathrm{ATT}=P$. attenuata, ATT $1=P$. sp. attenuata-like 1 , ATT $2=P$. sp. attenuata-like 2, ATT $3=P$. sp. attenuata-like 3, BOT2 $=P$. sp. botryosa-like 2 , CAS $=P$. castaneae, CHL $=P$. chlamydospora, $\mathrm{CIN}=$ P. cinnamomi, $\mathrm{CIT}$ VII $=P$. citricola $\mathrm{VII}, \mathrm{CIT} \mathrm{IX}=P$. citricola $\mathrm{IX}, \mathrm{CIT} \mathrm{X}=$ P. citricola $\mathrm{X}, \mathrm{CIT} \mathrm{XI}=P$. citricola $\mathrm{XI}, \mathrm{GRE}=P$. gregata, $\mathrm{HEV}=P$. heveae, $\mathrm{MEA} 1=P$. $\mathrm{sp}$. meadii-like $1, \mathrm{MEA} 2=$ P. sp. meadii-like 2, MUV1 $=$. sp. multivesiculata-like $1, \mathrm{PAR}=$. parvispora, $\mathrm{RAM}=$ P. ramorum; $\mathrm{TRO} 2=P$. sp. tropicalis-like 2 , VIE $=$ Nothophytophthora vietnamensis. ${ }^{\mathrm{b}} \mathrm{Mating}$ types: $\mathrm{A} 1=$ forming oogonia only in dual cultures with A2 tester strains; A2 = forming oogonia only in dual cultures with A1 tester strains; A2ho = forming oogonia in dual cultures with A1 tester strains and in ageing single cultures. ${ }^{c}$ Pythium senticosum also isolated. $\mathrm{d}$ Phytopythium sp, also isolated. e Phytopythium vexans s.l. also isolated. $\mathrm{f}$ Phytopythium sp. 1 PB-2013 also isolated. ${ }^{\mathrm{g}}$ Fallen leaves collected from the ground. ${ }^{\mathrm{h}}$ Pythium intermedium also isolated. ${ }^{\mathrm{i}}$ Pythium $\mathrm{sp}$. conidiophorum-like also isolated. ${ }^{\mathrm{j}}$ Phytopythium chamaehyphon also isolated. ${ }^{\mathrm{k}}$ Phytopythium cucurbitacearum also isolated. ${ }^{1}$ Phytopythium vexans also isolated. ${ }^{\mathrm{m}}$ Phytopythium sp. Côn Đảo also isolated. 
Table 2. Location and altitude of the 16 riparian sites sampled in spring 2016 and 2017 in Vietnam and Phytophthora and other oomycete taxa isolated.

\begin{tabular}{|c|c|c|c|c|c|c|}
\hline Site no. & $\begin{array}{c}\text { GPS } \\
\text { Coordinates }\end{array}$ & $\begin{array}{l}\text { Altitude (m } \\
\text { a.s.l) }\end{array}$ & River; Province & Location of Catchment and Vegetation & Sampling Method a & $\begin{array}{l}\text { Phytophthora and } \\
\text { Nothophytophthora spp. }{ }^{\mathrm{b}, \mathrm{c}}\end{array}$ \\
\hline R01 & $\begin{array}{l}\text { N22 } 20.127 \\
\text { E103 } 46.782\end{array}$ & 2083 & Forest stream 1; Lào Cai & $\begin{array}{l}\text { Hoàng Liên NP; subalpine and montane } \\
\text { Rhododendron scrub and forests, montane } \\
\text { broadleaved forests }\end{array}$ & $\begin{array}{c}\text { Baiting raft } \\
\text { Fallen leaves/flowers }\end{array}$ & $\begin{array}{l}\text { CAP, } \times \text { HET A1, } \times \text { HET A1ho, } \\
\text { MUV1, RAM A1 d } \\
\text { CIT VII, RAM A1, SYL2, VIE }\end{array}$ \\
\hline $\mathrm{R} 02$ & $\begin{array}{l}\text { N22 } 20.440 \\
\text { E103 } 46.576\end{array}$ & 2007 & Forest stream 2; Lào Cai & $\begin{array}{l}\text { Hoàng Liên NP; subalpine and montane } \\
\text { Rhododendron scrub and forests, montane } \\
\text { broadleaved forests }\end{array}$ & $\begin{array}{c}\text { Baiting raft } \\
\text { Fallen leaves/flowers }\end{array}$ & $\begin{array}{l}\text { RAM A1, SYL2 } \\
\text { GAL1, GAL2, MUV1, RAM A1, } \\
\text { SYL2, VIE e,f,g,h }\end{array}$ \\
\hline R03 & $\begin{array}{l}\text { N22 } 21.046 \\
\text { E103 } 46.273\end{array}$ & 1913 & $\begin{array}{l}\text { Forest stream 3, tributary } \\
\text { of forest stream 5; Lào Cai }\end{array}$ & Hoàng Liên NP; montane broadleaved forests & Baiting raft & $\begin{array}{l}\text { CHL, CIT VII, RAM A1, RAM } \\
\text { A2, SYL2 e }\end{array}$ \\
\hline R04 & $\begin{array}{l}\text { N22 } 21.029 \\
\text { E103 } 46.317\end{array}$ & 1904 & $\begin{array}{l}\text { Forest stream 4, tributary } \\
\text { of forest stream 5; Lào Cai }\end{array}$ & Hoàng Liên NP; montane broadleaved forests & Baiting raft & $\begin{array}{c}\text { CHL, } \times \text { HET A1, RAM A1, RAM } \\
\text { A2, SYL2, SYL3 }{ }^{\mathrm{i}}\end{array}$ \\
\hline R05 & $\begin{array}{l}\text { N22 } 20.906 \\
\text { E103 } 46.197\end{array}$ & 1895 & $\begin{array}{l}\text { Forest stream 5, Gold } \\
\text { river, downstream of R03, } \\
\text { R04, R06-R08; Lào Cai }\end{array}$ & Hoàng Liên NP; montane broadleaved forests & Baiting raft & $\begin{array}{c}\text { CHL, CIT VII, } \times \text { HET A1, RAM } \\
\text { A1, SYL2 e }\end{array}$ \\
\hline R06 & $\begin{array}{l}\text { N22 } 20.911 \\
\text { E103 } 46.199\end{array}$ & 1896 & $\begin{array}{l}\text { Forest stream 6, tributary } \\
\text { of forest stream 5; Lào Cai }\end{array}$ & Hoàng Liên NP; montane broadleaved forests & Baiting raft & CHL, $\times$ HET A 1 h,i \\
\hline R07 & $\begin{array}{l}\text { N22 } 20.902 \\
\text { E103 } 46.261\end{array}$ & 1912 & $\begin{array}{l}\text { Forest stream 7, tributary } \\
\text { of the Gold river; Lào Cai }\end{array}$ & Hoàng Liên NP; montane broadleaved forests & Baiting raft & $\times$ HET A1, RAM A1 \\
\hline R08 & $\begin{array}{l}\text { N22 } 20.904 \\
\text { E103 } 46.259\end{array}$ & 1911 & $\begin{array}{l}\text { Forest stream 5, Gold } \\
\text { river; Lào Cai }\end{array}$ & Hoàng Liên NP; montane broadleaved forests & Baiting raft & CIT VII, SYL2, SYL3 \\
\hline R09 & $\begin{array}{l}\text { N22 } 18.597 \\
\text { E103 } 52.426\end{array}$ & 1013 & $\begin{array}{c}\text { Muong Hoa River; Lào } \\
\text { Cai }\end{array}$ & $\begin{array}{l}\text { Hoàng Liên NP; subalpine and montane } \\
\text { Rhododendron scrub and forests, montane } \\
\text { broadleaved forests, rice fields }\end{array}$ & Baiting raft & $\mathrm{KEL}, \mathrm{PSC}, \times \mathrm{KUN}$ \\
\hline R10 & $\begin{array}{l}\text { N22 } 19.372 \\
\text { E103 } 49.780\end{array}$ & 1193 & $\begin{array}{l}\text { Forest stream 9, Cat Cat } \\
\text { River; Lào Cai }\end{array}$ & Hoàng Liên NP; montane broadleaved forests & $\begin{array}{c}\text { Baiting raft } \\
\text { Fallen leaves/flowers }\end{array}$ & $\begin{array}{c}\text { CAP, CIT VII, CIT VIII, SYL1, } \\
\text { SYL3 e } \\
\text { CHL, PSC, QUI, SYL 1, SYL3, } \\
\text { RAM A1, VIE f }{ }^{\mathrm{j}, \mathrm{k}}\end{array}$ \\
\hline R11 & $\begin{array}{l}\text { N22 } 22.230 \\
\text { E103 } 52.615\end{array}$ & 1308 & $\begin{array}{l}\text { Forest stream 8; tributary } \\
\text { of Ngòi Duôi River; Lào } \\
\text { Cai }\end{array}$ & $\begin{array}{l}\text { Sau Chua mountain; Chamaecyparis hodginsii } \\
\text { forest F24; broadleaved mountain forests and } \\
\text { Cunninghamia lanceolata plantations }\end{array}$ & $\begin{array}{c}\text { Baiting raft } \\
\text { Fallen leaves/flowers }\end{array}$ & $\begin{array}{l}\text { BIT, CIT VII, CIT IX, MAC, QUI, } \\
\text { SYL3 } \\
\text { CIT IX, KEL, RAM A1 }{ }^{1}\end{array}$ \\
\hline
\end{tabular}


Table 2. Cont.

\begin{tabular}{|c|c|c|c|c|c|c|}
\hline Site no. & $\begin{array}{c}\text { GPS } \\
\text { Coordinates }\end{array}$ & $\begin{array}{l}\text { Altitude (m } \\
\text { a.s.l) }\end{array}$ & River; Province & Location of Catchment and Vegetation & Sampling Method ${ }^{a}$ & $\begin{array}{c}\text { Phytophthora and } \\
\text { Nothophytophthora spp. }{ }^{\mathrm{b}, \mathrm{c}}\end{array}$ \\
\hline R12 & $\begin{array}{l}\text { N22 } 16.787 \\
\text { E104 } 13.394\end{array}$ & 63 & $\begin{array}{l}\text { Red River (Sông Hồng); } \\
\text { Lào Cai }\end{array}$ & $\begin{array}{l}\text { Large catchment in N-Vietnam and Yunnan; } \\
\text { subalpine and montane Rhododendron scrub } \\
\text { and forests, montane broadleaved forests, forest } \\
\text { plantations, rice fields, horticulture }\end{array}$ & Baiting raft & $\times \mathrm{KUN}, \times \mathrm{PER} 4, \times \mathrm{VIR}$ \\
\hline R13 & $\begin{array}{l}\text { N21 } 03.275 \\
\text { E105 24.050 }\end{array}$ & 59 & Stream 9; Hanoi & $\begin{array}{l}\text { Ba Vi NP; subtropical evergreen forests, rice } \\
\text { fields }\end{array}$ & Baiting raft & $\begin{array}{c}\times \mathrm{INS}, \times \mathrm{GRE} 3, \times \mathrm{KUNN}, \times \text { PER4, } \\
\times \mathrm{VIR}^{\mathrm{e}}\end{array}$ \\
\hline R14 & $\begin{array}{l}\text { N21 } 3.261 \\
\text { E105 } 24.012\end{array}$ & 60 & $\begin{array}{l}\text { Stream 10, tributary of } \\
\text { stream 9; Hanoi }\end{array}$ & $\begin{array}{l}\text { Ba Vi NP; subtropical evergreen forests, rice } \\
\text { fields }\end{array}$ & Baiting raft & $\times \mathrm{KUN}, \times \operatorname{PER} 4^{\mathrm{i}}$ \\
\hline R15 & $\begin{array}{l}\text { N21 } 06.177 \\
\text { E105 } 19.267\end{array}$ & 26 & $\begin{array}{l}\text { Black River (Sông Đà); } \\
\text { Hanoi }\end{array}$ & $\begin{array}{l}\text { Large catchment in N-Vietnam and Yunnan; } \\
\text { subalpine and montane Rhododendron scrub } \\
\text { and forests, montane broadleaved forests, } \\
\text { subtropical evergreen forests, forest plantations, } \\
\text { rice fields, horticulture }\end{array}$ & Baiting raft & DRE A1, $\times$ VIR $^{1, m}$ \\
\hline R16 & $\begin{array}{l}\text { N21 } 01.576 \\
\text { E105 27.218 }\end{array}$ & 26 & Stream 11; Hanoi & Forest plantations, rice fields, horticulture & Baiting raft & $\times$ PER $4, \times$ VIR $^{\mathrm{n}}$ \\
\hline \multicolumn{7}{|c|}{ 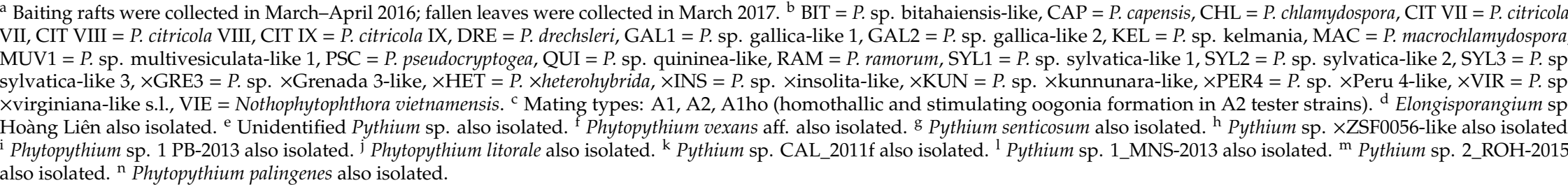 } \\
\hline
\end{tabular}


In total, nine previously unknown Phytophthora species from four of the five subclades within Clade 2 were detected in forest stands. From Clade 2a, P. sp. meadii-like 1 was isolated from the montane A. nepalensis stand F13 at $1717 \mathrm{~m}$ a.s.l. on Xin Chài mountain, while P. sp. meadii-like 2 was found in the tropical lowland rainforest stand F20 in Cuc Phuong NP (Figure 1; Table 1). The ITS sequences of all isolates of $P$. sp. meadii-like 1 were identical except for one isolate with an extra $\mathrm{T}$ in position 11 and an $\mathrm{A}$ instead of a $\mathrm{T}$ in position 12 (Supplementary Table S1). This new taxon differed in the ITS from P. meadii (isolate P75; GenBank no. GU993903) at positions 137 and 632 which were shared with the ex-type isolate of P. botryosa (CBS586.69; GenBank no. HQ643151), and from P. botryosa at five positions $(72,152,444,460,773)$ which were identical with $P$. meadii. In addition, all isolates of $P$. sp. meadii-like 1 had a unique deletion at position 146 . The ITS sequences of $P$. sp. meadii-like 2 showed intraspecific variability at positions $11,13,22$. Most isolates differed from $P$. sp. meadii-like 1 , $P$. meadii isolate P75 and the ex-type isolate of $P$. botryosa by having four unique heterozygous positions $(161,444,502,713)$. In addition, $P$. sp. meadii-like 2 showed in the ITS the same differences to P. meadii and $P$. botryos $a$ as $P$. sp. meadii-like 1 . The ITS sequences of both new taxa showed differences to the ex-type isolate of the recently described P. mekongensis from southern Vietnam (CBS135136; GenBank no. KC875838) at eight positions $(152,155,163,165,166,175,179,750)$. A third new taxon from Clade 2a, P. sp. botryosa-like 2, was exclusively isolated from the tropical lowland rainforest stand F25 on Côn Đảo island (Figure 1; Table 1). The ITS sequences of all isolates were identical to each other and differed from P. botryosa and P. meadii at four $(72,137,161,460)$ and five positions $(152,161$, $444,632,773)$, respectively. In a 610 bp alignment of cox1, P. sp. meadii-like $1, P$. sp. meadii-like 2 and P. sp. botryosa-like 2 differed from P. meadii (isolate p75; GU945489) at 14, 13 and 12 positions, respectively, and from $P$. botryosa (HQ261256) at 10, 9, and 8 positions, respectively. According to sequence analyses, the closest relatives of $P$. sp. botryosa-like 2 were an isolate obtained in 1930 from Cocos nucifera in Sulavesi (CBS235.30) which differed in ITS (HQ643140) by five heterozygous positions and in cox1 (HQ708214) at five positions, and an isolate of unknown origin which was obtained from a vanilla plant in 1928 (CBS238.28) and showed differences at five positions in both ITS (HQ643139) and cox1 (HQ708213) of which three were heterozygous in ITS. All isolates of $P$. sp. botryosa-like 2, P. sp. meadii-like 1 and $P$. sp. meadii-like 2 produce caducous papillate sporangia with variable shapes and are heterothallic, exclusively belonging to mating type A1. Oospore abortion rates in mating tests with A2 tester strains of P. meadii and P. botryosa exceeded 95\%.

From the montane A. nepalensis stand F13 a new Phytophthora species from Clade 2b was isolated which differed from the ex-type isolate of P. tropicalis (CBS434.91) in ITS (HQ643369) and cox1 (HQ708417) at 5 and 7 positions, respectively, and is hence informally designated as $P$. sp. tropicalis-like 2. Similar to $P$. tropicalis, all isolates produce thickwalled chlamydospores and papillate sporangia. Phytophthora sp. tropicalis-like 2 differs from $P$. tropicalis [49] by producing sporangia which are only partially caducous and have shorter pedicels $(24.7 \pm 16.8 \mu \mathrm{m}$ vs. $>50 \mu \mathrm{m})$ and shorter length/breath $(\mathrm{l} / \mathrm{b})$ ratio $(1.8 \pm 0.3$ vs. $1.8-2.4)$.

Phytophthora citricola VII, informally designated from a mountain forest in Taiwan [10], and another three new taxa from the 'P. citricola complex' in Clade 2c, informally designated here as P. citricola IX, P. citricola X and P. citricola XI, were isolated from the montane A. nepalensis stand F13, the subtropical evergreen forest stand F16 and the tropical lowland rainforest stands F21 and F25, respectively (Figure 1; Table 1). Phytophthora citricola VII, IX, X and XI differ from the authentic type of P. citricola s.s. (CBS295.29; ITS-FJ560913; cox1-KC855432) in the ITS (771 bp alignment) at 3, 3, 12 and 11 positions, and in cox1 (1231 bp alignment) at 23, 19, 29, and 15 positions, respectively. Like other members of the 'P. citricola complex', the four new species are homothallic forming smooth-walled oogonia with paragynous antheridia. The sporangia of $P$. citricola VII and IX resemble those produced by other species from Clade 2c in being semipapillate, persistent and with exclusively external proliferation. In contrast, P. citricola $\mathrm{X}$ and XI produce mainly papillate sporangia with both external and, infrequently, also internal extended and nested proliferation. In addition, P. citricola $\mathrm{X}$ is distinguished from all known 
related species by forming abundant catenulate hyphal swellings in water. Phytophthora citricola VII produces a high proportion of zoospores with a ring-like to oval coiling of both flagella ends.

From a swampy depression in the montane evergreen cloud forest F06 in Hoàng Liên NP, a previously unknown Phytophthora species from Clade 2e was isolated which is provisionally named as P. sp. multivesiculata-like 1. Its ITS and cox 1 sequences differ from the ex-type isolate of P. multivesiculata (CBS545.96; HQ643288 and HQ708340) at eight and 38 positions, respectively. The ITS sequences of two yet undescribed species, Phytophthora sp. aquatilis (GenBank no. FJ666126) and Phytophthora sp. Costa Rica 5 (KC479200), show differences to $P$. sp. multivesiculata-like 1 at 8 and 6 positions, respectively. Like $P$. multivesiculata, $P$. sp. multivesiculata-like 1 is homothallic with aplerotic oospores and produces in water numerous catenulate hyphal swellings and both nonpapillate and semipapillate sporangia with external and internal proliferation. However, it can easily be distinguished from P. multivesiculata [50] by forming considerably larger sporangia (on av. $57.2 \times 32.8$ vs. $45 \times 33 \mu \mathrm{m}$ ), larger oogonia ( $45 \mathrm{vs} .41 \mu \mathrm{m}$ ) with highly variable shapes ranging from globose, excentric or elongated with long tapering bases to comma-shaped, and exclusively amphigynous antheridia.

Also in Hoàng Liên NP, P. gregata from Clade $6 \mathrm{~b}$ was recovered from the rhizosphere of Meliosma henryi and Neolitsea merilliana in the montane evergreen cloud forest F06 while the other Clade 6b species $P$. chlamydospora and P. ramorum from Clade $8 \mathrm{c}$ were isolated from fallen leaves of $R$. arboreum collected from the forest ground in the montane, evergreen broadleaved forest F11 (Table 1).

Besides the recently described Nothophytophthora vietnamensis [51] which was isolated from the rhizosphere of C. acuminatissima and Acer campbellii in the montane evergreen cloud forest F04 and A. nepalensis in stand F13 on Xin Chài mountain, a range of Pythium and Phytopythium species including Py. intermedium, Py. senticosum, Ph. chamaehyphon, Ph. cucurbitacearum, Ph. sp. 1 PB-2013, 14 haplotypes from the $P h$. vexans complex and two previously unknown taxa, informally designated as Py. sp. conidiophorum-like and Ph. sp. Côn Đảo, were obtained from 15 forest stands (Table 1 and Supplementary Table S1).

\subsection{Phytophthora Diversity in Natural Forest Streams and Rivers}

Using rafts with leaves of $C$. indica, C. sinensis, L. bacgangensis, Q. glauca, and, less frequently, Carpinus sp., C. hodginsii, Cinnamomum iners, Dipterocarpus alatus, Prunus sp., Q. gilva and A. mangium as in situ baits in all 16 rivers and streams tested, and freshly fallen leaves of different tree species and flowers of R. arboreum and R. leptocladus in four forest streams, seven known species (P. capensis, P. chlamydospora, P. drechsleri, P. macrochlamydospora, P. pseudocryptogea, P. ramorum, $P$. $\times$ heterohybrida), five informally designated taxa (P. citricola VII, P. sp. kelmania, $P$. sp. $\times$ insolita-like, $P$. sp. $\times$ kunnunara-like, P. sp. Xvirginiana-like s.l.) and 12 previously unknown taxa of Phytophthora were isolated (Table 2). The latter included $P$. sp. multivesiculata-like 1, two new species from the 'P. citricola complex', three and one new species related to the Clade 6 taxa $P$. sp. sylvatica and $P$. sp. bitahaiensis, respectively, three new species from Clade 9 and two new species related to $P$. gallica from Clade 10.

The Phytophthora communities in the 11 montane streams above $1000 \mathrm{~m}$ a.s.l. with a temperate climate were dominated by species belonging to Clades 2, 6, 7, and 8 whereas from the five lowland rivers with subtropical to tropical climate almost exclusively Phytophthora species from Clade 9 were obtained (Figure 1; Table 2).

In montane streams, the most widespread species was P. ramorum which could be recovered from seven of the eight forest streams above $1890 \mathrm{~m}$ altitude in the Fansipan area and in 8-12 km distance to these sites from stream R11 originating from the C. hodginsii forest F24 at Sau Chua mountain in $1300 \mathrm{~m}$ altitude. Both mating types were obtained with the A1 mating type occurring in eight streams and the A2 in two streams. In the latter (streams R03, R04) both mating types co-occurred (Table 2). In the two streams (R01, R02) sampled in both 2016 and 2017 only mating type A1 was isolated. The 65 P. ramorum isolates exhibited five slightly different ITS genotypes. Eight isolates from four streams (R02, R04, R05, R10) were identical to the ex-type isolate from Germany, which belongs to the EU1 lineage (CBS101553; HQ643339). The most common genotype (46 isolates) differed from the ex-type by 
having a T instead of a Y at position 616, while three isolates from three streams (R01, R02, R10) had a $\mathrm{C}$ at this position. Four P. ramorum isolates from streams R02 and R03 were distinguished from the ex-type by being heterozygous at position 682 ( $R$ instead of $G$ ) while in one isolate from stream R03 both heterozygous positions occurred. For 43 isolates, representative for all eight streams, forest site F11 and both mating types, cox1 was sequenced and compared to representative isolates of the four known P. ramorum lineages EU1, EU2, NA1, and NA2. In a 1240 bp long cox1 alignment all but one of the Vietnamese P. ramorum isolates were identical and differed from four representative isolates of the North American NA2 lineage only at position 123. They differed from EU1 by 5 bp (positions 123, $808,1141,1156,1202)$, EU2 by 5 bp $(624,966,1035,1156,1240)$ and NA1 by 4 bp $(123,808,1156,1202)$, respectively. Isolate VN88 differed from the other 42 isolates by having a unique polymorphism at position 1228 (C instead of A). The morphological structures of all isolates were congruent with the original description of P. ramorum [52].

From Clade 2c, P. capensis, P. citricola VII, P. citricola VIII, and P. citricola IX were isolated from two, one, five, and one montane forest streams, respectively (Table 2). From stream R11, P. capensis, and P. citricola VII and VIII were obtained while in another two streams (R01, R10) two different species from the 'P. citricola complex' co-occurred. Compared to the ex-type isolate of P. capensis (P1819; ITS-GU191232; cox1-GU191275) from South Africa, the ITS sequences of the Vietnamese isolates were identical but their cox 1 sequences were separated in a 598 bp alignment by 9 bp (1.5\%). Phytophthora citricola VIII differed from the authentic type isolate of P. citricola s.s. (CBS295.29; ITS-FJ560913; cox1-KC855432) and from $P$. citricola VII, IX, X and XI in ITS (771 bp alignment) at 2, 2-3, 4, 12 and 11 positions, and in cox1 (1231 bp alignment) at 33, 26, 35, 42, and 37 positions. Being homothallic with paragynous antheridia and producing semipapillate sporangia of variable shapes, P. citricola VIII morphologically resembles other species from Clade 2c.

Phytophthora sp. multivesiculata-like 1 from Clade 2e was present in streams R01 and R02 which originate from a catchment area around forest stand F06 where this new taxon was also found.

From Clade 6b, which contains numerous predominantly aquatic Phytophthora species, $P$. chlamydospora, three new species informally designated as $P$. sp. sylvatica-like 1,2 and 3 , and another new species designated as $P$. sp. bitahaiensis-like were recovered from 8 of the 11 mountain streams (Table 2). Most common were P. sp. sylvatica-like 2 (six streams), P. chlamydospora (5 streams) and $P$. sp. sylvatica-like 3 (four streams). In five streams more than one Clade $6 \mathrm{~b}$ species were found. In the ITS (844 bp alignment) and cox1 (861 bp alignment), P. sp. sylvatica-like 1 differs from its closest relative $P$. sp. forestsoil-like from Taiwan (KU682574) at 2 and 7 positions, respectively, while $P$. sp. sylvatica-like 2 and 3 show differences to $P$. sp. forestsoil-like in ITS at 12-13 and 11-12 positions, respectively, and in cox 1 at 29 and 27 positions. Phytophthora sp. sylvatica-like 1 differs from $P$. sp. sylvatica-like 2 and 3 in ITS by 9-11 and 7-8 bp, respectively, and in cox 1 by 44 and 46 bp, respectively. The latter two species can be distinguished in the ITS and $\operatorname{cox} 1$ by differences at $2-4$ and 11 positions, respectively. Phytophthora sp. bitahaiensis-like differs in the ITS from $P$. sp. bitahaiensis (isolate BHL1; KT183432) from a forest stream in Yunnan, China, at 4 positions. Unfortunately, for $P$. sp. bitahaiensis no cox 1 sequences are available. Similar to $P$. sp. forestsoil-like and many other aquatic Clade 6 species [10,21,53], P. sp. sylvatica-like 1, 2, and 3 and $P$. sp. bitahaiensis-like are sterile and form abundantly nonpapillate sporangia with internal nested and extended proliferation. All ten sequenced isolates from $P$. chlamydospora were identical in both the ITS and cox 1 and differed from the ex-type isolate of P. chlamydospora from the UK (P236; AF541900, MH136867) only by being in the ITS heterozygous at position 57 ( $R$ instead of $G$ ) while being identical to the ex-type in cox 1 .

From five mountain streams above $1890 \mathrm{~m}$ altitude, the recently described Clade 7 a hybrid species P. $\times$ heterohybrida was isolated. All 12 isolates differed in the ITS from the ex-type isolate from Taiwan (CBS141207; KU517151) at position 77 (Y instead T). In addition, three isolates from stream R01 were separated from the ex-type by being homozygous at position 428 ( $\mathrm{T}$ instead of $\mathrm{Y}$ ) and by having two unique heterozygous positions (656 and 748). In a $876 \mathrm{bp}$ alignment of cox 1 , one isolate from stream R01 differed at three positions from the ex-type isolate (KU517145) and from all other Vietnamese and 
Taiwanese isolates which were identical. Mating tests with A1 and A2 tester strains of P. ×heterohybrida from Taiwan showed that all isolates from Vietnam belonged to the A1 mating type. In addition, two isolates from stream R01 produced oogonia abundantly in single culture. The morphology of the ornamented oogonia, mostly two-celled amphigynous antheridia and nonpapillate sporangia of the Vietnamese isolates matched the original description of $P$. $\times$ heterohybrida [22].

Phytophthora pseudocryptogea and P. sp. kelmania from Clade 8a were isolated from each two of the three lower montane streams R09-R11 (Table 2). The three isolates of P. pseudocryptogea from streams R09 and R10 differed in ITS (816 bp alignment) and cox1 (672 bp alignment) from the Australian ex-type isolate (VHS16118; KP288376, KP288342) at three and one positions, respectively. The five isolates of $P$. sp. kelmania from streams R09 and R11 showed differences in ITS (841 bp alignment) and cox1 (582 bp alignment) to isolate P10614 (HQ261691, HQ261438) from North America at 3, 4, and 5 positions, respectively. The morphology of all Vietnamese isolates of P. pseudocryptogea and P. sp. kelmania matched the descriptions in literature [54].

From forest stream R02 in 2007 m altitude two new species from Clade 10 were isolated from naturally fallen leaves. Phytophthora sp. gallica-like 1 and $P$. sp. gallica-like 2 were distantly related to $P$. gallica differing in a $885 \mathrm{bp}$ alignment of the ITS from the ex-type isolate of the latter (CBS 111474 = GAL1; DQ286726) at 53 and 68 positions, respectively, while being separated from each other by $49 \mathrm{bp}$. Morphologically, both new species can easily be distinguished from the sterile P. gallica [55] by being homothallic forming smooth-walled oogonia with paragynous antheridia. Phytophthora sp. gallica-like 1 produces globose chlamydospores like P. gallica whereas P. sp. gallica-like 2 does not form chlamydospores.

From Clade 9a2, P. macrochlamydospora and a new species related to P. quininea, informally designated as $P$. sp. quininea-like, co-occurred in montane forest stream R11. Isolate VN1006 of P. macrochlamydospora differed in the ITS (816 bp alignment) and in cox1 (670 bp alignment) from the Australian ex-type isolate (P10263; FJ801351, MH136923) at 2 and 3 positions, respectively. In accordance with the original description of P. macrochlamydospora [2], the Vietnamese isolate was sterile and produced large chlamydospores and semipapillate to non-papillate sporangia. The four isolates of $P$. sp. quininea-like were separated in the ITS and cox 1 from the ex-type isolate of $P$. quininea (CBS407.48 = P8488; HQ261660; AY564200 + HQ708386) from Peru by differences of 7 and 27 bp, respectively. Like $P$. quininea, $P$. sp. quininea-like produces non-papillate sporangia with internal and external proliferation, catenulate irregular hyphal swellings and thick-walled large chlamydospores. Interestingly, two of the four isolates were sterile while the other two isolates were homothallic like P. quininea [2] but can be distinguished from the latter by forming amphigynous instead of paragynous antheridia. Only one isolate of the Clade 9a1 hybrid taxon $P$. sp. $\times$ kunnunara-like could be obtained from one lower montane stream (R09) in Hoàng Liên NP (Table 2).

In contrast to the montane streams, the Phytophthora communities in the five lowland rivers with subtropical to tropical climate were dominated by Phytophthora taxa from the high-temperature tolerant Clades 9a1 and 9a3. From Clade 9a1 potential hybrid isolates related to P. virginiana were obtained from the Red River, the Black River and two other streams (R13, R16) (Table 2). The potential hybrids differed in the ITS from the ex-type isolate of P. virginiana (46A2; KC295544) by having in total 10 heterozygous positions, with 1-8 heterozygous positions per isolate, which are partly not present in the three hybrid taxa $P$. sp. ×virginiana-like 1, 2, and 3 from Taiwan. Therefore, these Vietnamese isolates are informally designated as $P$. sp. $\times$ virginiana-like sensu lato. Phytophthora sp. $\times$ kunnunara-like was found in the Red River and the two streams originating from Ba Vì NP (R13, R14) (Table 2). Compared to P. sp. kunnunara from Western Australia, the ITS of the Vietnamese isolates had 10 heterozygous positions, with 1-8 heterozygous positions per isolate, which were only partly shared with Taiwanese isolates of $P$. sp. $\times$ kunnunara-like (KU682602, KU682603). Another swarm of potential hybrid isolates was abundantly obtained from four of the five lowland streams. They differed in the ITS from P. sp. Peru 4 (KC479209) at 10 positions which were all heterozygous (1-6 per isolate) and are, hence, informally designated as $P$. sp. $\times$ Peru 4-like. Finally, isolates of another new potential 
hybrid taxon, $P$. sp. $\times$ Grenada 3-like were recovered from stream R13 which were distinguished in the ITS from $P$. sp. Grenada 3 (KC479208) by having five instead of one heterozygous position. All isolates of $P$. sp. $\times$ Grenada 3-like, $P$. sp. $\times$ kunnunara-like, $P$. sp. $\times$ Peru 4-like and $P$. sp. $\times$ virginiana-like were in culture fast-growing, self-sterile and produced intercalary or laterally globose, club-shaped to irregular hyphal swellings, mostly globose thin-walled chlamydospores and nonpapillate sporangia with internal nested and extended proliferation, typical features of aquatic Clade 9 species [56].

Phytophthora sp. Xinsolita-like from Clade 9a3 was found in stream R13. Compared to the Taiwanese ex-type isolate of P. insolita (IMI288805; AF271222) the Vietnamese isolates showed differences in the ITS at eight positions of which four were heterozygous and mostly shared with Taiwanese isolates of $P$. sp. Xinsolita-like (KU682601). Morphologically, all isolates from stream R13 were similar to both $P$. insolita and P. sp. Xinsolita-like from Taiwan $[2,10]$, producing in single culture smooth-walled oogonia without antheridia, thin-walled chlamydospores and non-papillate sporangia with internal nested and extended proliferation.

The only Phytophthora species recovered from a lowland stream (Black River) and not belonging to Clade 9 was $P$. drechsleri from Clade 8a. The isolates differed from the ex-type isolate of $P$. drechsleri (ATCC 46724 = 23J5; AF266798, MH620076) in the ITS at one position (136; Y instead of T) and in a 862 bp alignment of $\operatorname{cox} 1$ at five positions. All three isolates belonged to the A1 mating type.

With 10, eight and six Phytophthora species, respectively, the montane forest streams R10, R11 and R02 harboured the highest diversity of Phytophthora species while the lowland rivers contained highly diverse assemblies of Clade 9 hybrids with almost all isolates being different from each other in the ITS.

Nothophytophthora vietnamensis was isolated from the montane forest streams R01, R02 and R10 in Hoàng Liên NP. In addition, the novel Elongisporangium sp. Hoàng Liên, Phytopythium litorale, Ph. vexans s.1., Ph. sp. 1 PB-2013, Py. senticosum, Py. sp. CAL_2011f, Py. sp. 1_MNS-2013, the previously unknown hybrid taxon $P y$. sp. $\times Z$ ZS0056-like and unidentified Pythium spp. were recovered from eight of the eleven mountain streams (Table 2 and Table S1). In four of the five lowland rivers, Ph. palingenes, Ph. sp. 1 PB-2013, Py. sp. 1_MNS-2013, Py. sp. 2_ROH-2015 and unidentified Pythium spp. were found (Table 2 and Table S1).

\subsection{Association between Phytophthora Presence in the Rhizosphere and Disease Symptoms}

In the 20 Phytophthora-inhabited forests sampled, the majority of the 52 tree species from which Phytophthora species were recovered appeared generally healthy (Figure 2a-f). Symptoms indicative of Phytophthora root diseases were almost exclusively found in eight montane forest stands and were mainly restricted to tree species belonging to the Ericaceae, Fagaceae and Lauraceae (Figures 3 and 4).

In Hoàng Liên NP, scattered dieback of Rhododendron arboreum trees with presence of $P$. cinnamomi A1 and $P$. sp. attenuata-like 1 in the rhizosphere was observed in the upper montane Rhododendron cloud forest at $2636 \mathrm{~m}$ altitude. In the montane evergreen cloud forest F03 at $2337 \mathrm{~m}$ altitude, groups of mature Quercus glauca trees showed severe thinning and dieback of the crowns (Figure 3a) which was associated with presence of P. cinnamomi A2 in the rhizosphere. In contrast, in forest stand F12 at 1895 $\mathrm{m}$ altitude, infested by P. cinnamomi A1, all Q. glauca trees appeared healthy. In six of the nine montane evergreen forest stands sampled between 1895 and $2242 \mathrm{~m}$ altitude, C. acuminatissima and sometimes also Neolitsea poilanei and N. polycarpa showed severe thinning and dieback of the crowns and mortality (Figure $3 b-d, f)$. Disease incidence was particularly high in the swampy depression sampled in stand F06 (Figure 3c,d,f). In seven and five of the nine stands, P. cinnamomi A2 and P. castaneae, respectively, were recovered from rhizosphere soil samples while P. cinnamomi A1, P. attenuata, P. gregata, P. sp. attenuata-like $1, P$. sp. attenuata-like 2 and $P$. sp. multivesiculata-like 1 were only infrequently found (Table 1). On a visual examination root samples from three declining trees each of C. acuminatissima and N. polycarpa, all infested by P. cinnamomi A2 and/or P. castaneae, exhibited severe losses of lateral and fine roots and open callussing lesions on coarse roots (Figure 4a-d). In stand F07, P. cinnamomi A2 was isolated from a bleeding bark lesion on a surface root of a declining C. acuminatissima tree (Figure 3e). 


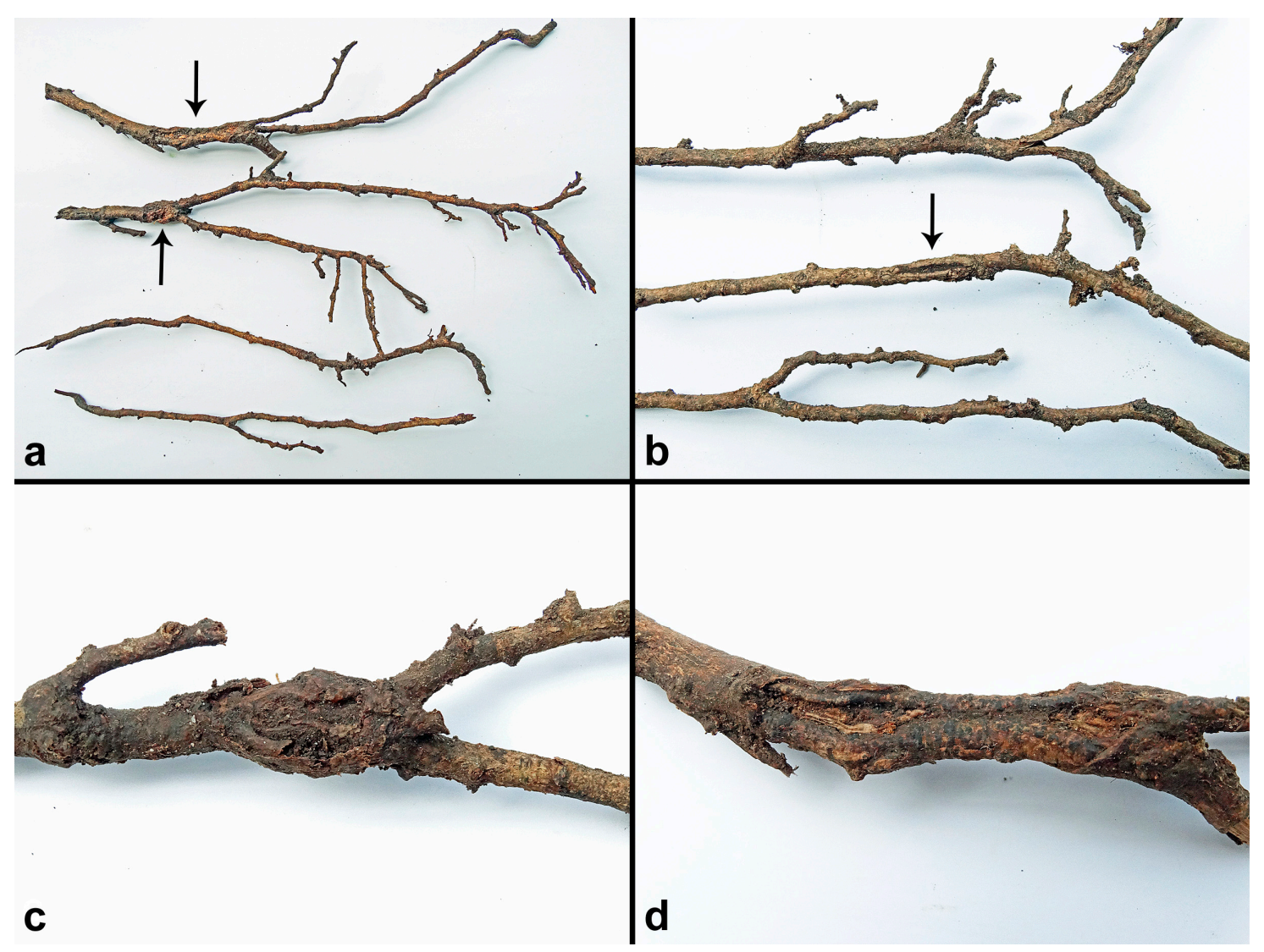

Figure 4. Symptoms on root systems of declining Neolitsea polycarpa trees in the montane evergreen cloud forest F10 in Hoàng Liên National Park associated with presence of P. cinnamomi A2 in the surrounding soil; $(\mathbf{a}, \mathbf{b})$ severe losses of lateral roots and fine roots and open callussing lesions on coarse roots (arrows); (c,d) detailed view of the open callussing lesions on the coarse roots from Figure 4a.

In contrast to the montane forests of Hoàng Liên NP, in the three submontane (700-1100 m a.s.1.) stands sampled in the subtropical, humid evergreen forests of Ba Vì NP all tree species, including several species from the Fagaceae genera Castanopsis, Lithocarpus and Quercus and the Lauraceae genera Litsea, Machilus and Phoebe, were healthy despite the occurrence of both mating types of P. cinnamomi and a range of five other Phytophthora species, including P. castaneae, P. heveae, P. parvispora, P. citricola IX and P. sp. attenuata-like 3 , in the soil. The only exception was a small patch dieback of Dysoxylum juglans with presence of $P$. sp. attenuata-like 3 in the rhizosphere (Figure $3 \mathrm{~g}$ ).

In the five Phytophthora-infested tropical lowland rainforest stands in Cuc Phuong (F18, F20, F21), Bù Gia Mập (F23), and Côn Đảo (F25) National Parks, no symptoms suggestive of Phytophthora diseases were observed.

\section{Discussion}

Vietnam harbours an extremely diverse flora probably due to its heterogeneous geology, geomorphology and climates and its transitional position between the eastern Himalayas, Yunnan, and the Indomalaysian archipelago on the Asian continental shelf [39-41]. The latter enabled repeated immigrations of plant and most likely also fungal and oomycete species during various glacial periods followed by subsequent speciations and species radiations in the interglacials. A similar scenario was proposed earlier for Taiwan [10,36-38]. We have shown here that the floristic and environmental diversity of Vietnam is reflected by the high diversity of oomycete taxa. In this survey of 25 natural forests and 16 rivers 13 described species, five informally designated taxa and 21 previously unknown taxa of Phytophthora, together with N. vietnamensis and a range of seven described and ten undescribed species of Elongisporium, Pythium and Phytopythium were obtained. Considering the relatively limited 
number and diversity of the sampled sites and ecosystem types it may be assumed that the true Phytophthora diversity of Vietnam is markedly higher. The finding of 20 Phytophthora taxa in 98 soil and four leaf samples from the 25 forest stands and an additional 15 Phytophthora taxa in 11 forest streams in Vietnam indicates a much higher diversity of forest Phytophthoras exists in Vietnam than occurs in Europe, the eastern US or the western US. In the latter areas, 39, 7, and 21 Phytophthora species, respectively, were detected in numerous surveys involving many more samples collected over much larger areas and a wider range of ecosystems [5,14,23,44,57-59].

The remote location of most sampled forest stands and forest streams in Vietnam, absence of introduced crop or tree species in the catchment areas and, apart from P. cinnamomi A2 in higher altitudes, the lack of association of Phytophthora with obvious disease symptoms suggest that most of the 35 forest Phytophthora species obtained are native to Vietnam. In contrast, only nine of the 32 Phytophthora species from European forests are considered indigenous [5,23,60]. The forest Phytophthora populations in Vietnam and Europe share only five species, P. chlamydospora, P. cinnamomi A2, P. pseudocryptogea, P. ramorum and P. sp. kelmania, while Vietnamese and North American forests have only P. chlamydospora, P. cinnamomi A2 and P. ramorum in common. Recent surveys in Taiwan, where floristic diversity is comparable to Vietnam, revealed a comparable Phytophthora diversity, with ten described and 17 previously unknown species from 30 forest stands and 25 streams [10,13]. Further, the Phytophthora communities revealed in Vietnam and Taiwan shared 12 taxa: P. attenuata, P. capensis, P. castaneae, P. chlamydospora, P. cinnamomi A1 and A2, P. citricola VII, P. heveae, P. parvispora, P. $\times$ heterohybrida, P. sp. ×insolita-like, P. sp. $\times$ kunnunara-like and $P$. sp. $\times$ virginiana-like s.l.. In three areas in northern Yunnan, a Chinese province adjacent to northern Vietnam, eight Phytophthora species were isolated from streams running through sclerophyllous oak forests but only two of them, $P$. chlamydospora and P. plurivora, were recovered from forest soil samples [16]. The only Phytophthora species common to Vietnam and northern Yunnan were P. chlamydospora and P. gregata. In montane forests of the tropical island Hainan, located in the South China Sea close to Vietnam, six Phytophthora species were found [12] of which three species, P. castaneae, P. cinnamomi and P. heveae, also occurred in Vietnam. The lower Phytophthora diversities in the north Yunnan and Hainan surveys compared to Vietnam were most likely due to the smaller number of sites and forest types sampled and the use of different isolation techniques.

In recent years, an impressive diversity of both known and previously unknown Phytophthora species has been revealed from stream surveys in several countries, including the eastern and western USA, Chile, Australia, South Africa and Taiwan [10,11,14,17,18,44,61], as discussed previously [10]. By comparison the riparian Phytophthora communities identified here in Vietnam are remarkably rich, with seven described species, five informally designated taxa and 12 previously unknown taxa. Several montane streams with small catchments in the forests around the Fansipan harboured an unprecedented diversity of up to ten Phytophthora species per stream.

Interestingly, the most common Phytophthora species in Vietnamese forest soils, P. cinnamomi, $P$. castaneae, $P$. heveae and the four species from the ' $P$. attenuata complex', were never isolated from streams running through or originating from infested forests. Overall, the Phytophthora communities found in the forest soils (20 taxa) and in the streams (24 taxa) shared only four species, P. chlamydospora, P. citricola VII and IX and P. sp. multivesiculata-like 1. Similar differences between terrestrial and aquatic Phytophthora populations were observed in comparable studies in Europe, Chile, Taiwan, South Africa and the USA $[10,11,18,44,58]$. This is consistent with previous observations that most Phytophthora species are adapted either to a soilborne and root-infecting or aerial foliage-infecting lifestyle, or are aquatic saprotrophs that tend to be opportunistic pathogens $[6,10,11,21,53,62]$. Consequently, when sampling Phytophthora diversity in a diverse environment both soils and streams should be analysed using optimal baiting methods for each or metagenomic approaches based on high-throughput pyrosequencing of environmental DNA with Phytophthora-specific primers [19,63]. Ideally, because metagenomic analyses can sometimes result in false molecular operational taxonomic units (MOTUs), 
and because living isolates are needed for taxonomic descriptions and host range testing, baiting, and metagenomic approaches should be carried out in parallel.

Altitude had a strong influence on Phytophthora distribution. The 'P. attenuata complex', P. castaneae and $P$. cinnamomi occurred only in soils of submontane and montane forests above $700 \mathrm{~m}$ a.s.l. while $P$. heveae and most taxa from Clade 2a were restricted to forests below $1100 \mathrm{~m}$ altitude. The altitudinal influence on aquatic Phytophthoras was even more pronounced. While the 11 montane streams above $1000 \mathrm{~m}$ altitude with a subtropical to temperate climate contained mainly species belonging to Clades $2 \mathrm{c}, 2 \mathrm{e}, 6 \mathrm{~b}, 7 \mathrm{a}$, and $8 \mathrm{c}$, the Phytophthora communities in the five lowland rivers with subtropical to tropical climate were dominated by species and hybrids from the high-temperature tolerant Clades 9a1 and 9a3 [28]. Clade 9 species and hybrids were also most common in lowland streams in Taiwan and South Africa $[10,18]$.

The results of this survey offer new insights into the origin of several invasive Phytophthora pathogens and of clades and subclades of Phytophthora. Most notably, the finding of the highly invasive, wide-host range pathogen P. ramorum in eight forest streams around the Fansipan and Sau Chua mountains with both A1 and A2 mating types present, together with an apparent absence of overtly visible disease symptoms on potentially susceptible Ericaceae, Fagaceae, or Lauraceae, suceptible genera where P. ramorum is damaging and introduced in Europe and North America [6,7,64], suggests an equilibrium between the pathogen and the north Vietnamese vegetation as a consequence of long term endemism and co-evolution. This is supported by variability in the ITS and cox1 sequences of the Vietnamese isolates and by cox 1 sequence differences between the Vietnamese isolates and the North American NA1 and NA2 and the European EU1 and EU2 lineages. Because of the implications both for the origin of the pathogen and for international biosecurity a detailed comparative phenotypic and molecular analysis of the Vietnamese P. ramorum isolates and the known EU1, EU2, NA1, and NA2 lineages [65] is currently ongoing to further characterise the Vietnamese population and its relationship to the known lineages. Since southern Yunnan, northern Laos, and the eastern Himalayas belong to the same biogeographic area as the Fansipan region mountain forests in these regions may also harbour endemic $P$. ramorum populations. Further surveys are needed to confirm this hypothesis.

Phytophthora cinnamomi was the most common soilborne Phytophthora species above $700 \mathrm{~m}$. The A2 mating type of P. cinnamomi was more widespread, occurring in 11 forest stands between 713 and 2337 $\mathrm{m}$, whereas the A1 occurred only in four forest stands located between 1108 and $2636 \mathrm{~m}$ a.s.1. In Taiwan and Papua New Guinea also the A1 mating type occurrs at higher altitudes than the A2 indicating higher tolerance to low temperatures $[10,66]$. However, in both of these locations the altitudinal differences between the mating types are larger than in Vietnam, the A2 being confined to the lowland forests. In each one stand in Hoàng Liên NP and Ba Vì NP both mating types co-occurred. In Hoàng Liên NP the A1 was present in the upper montane Rhododendron forest at $2636 \mathrm{~m}$ and in two lower montane stands at $1900 \mathrm{~m}$. However, it was not detected in the eight forest stands between 2337 and $2022 \mathrm{~m}$ in which not only the A2 type was present but severe dieback of Fagaceae and Lauraceae was observed (notably C. acuminatissima, Q. glauca, N. poilanei and N. polycarpa). Pathogenicity trials are required to fulfill Koch's postulates for these host-pathogen associations and confirm that P. cinnamomi $\mathrm{A} 2$ is causing the dieback of these native Fagaceae and Lauraceae species. In contrast, no dieback was observed in the three forest stands in Ba Vì NP between 713 and $1100 \mathrm{~m}$, despite the presence of P. cinnamomi A2. In the two stands with the co-occurrence of both mating types the A1:A2 ratio of the 44 isolates was 59.1:40.9, whereas the overall mating type ratio of the 151 isolates from $13 P$. cinnamomi infested stands was 30.5:69.5. Collectively, these results suggest that, as a consequence of current climatic warming, the more thermophilic but frost sensitive A2 mating type may be spreading into higher altitudes in Vietnam. Such a progression of P. cinnamomi A2 into higher latitudes and altitudes with climate change was predicted by CLIMEX modelling [67-69]. In the newly A2 invaded high-altitude forests in Vietnam the A2 may be outcompeting and replacing the native co-evolved A1, causing dieback in the susceptible non-coevolved hosts. The widespread distribution of P. cinnamomi in northern Vietnam, the co-occurrence of both mating types in several stands, and the absence 
of disease symptoms in lower altitudes also indicates that Vietnam lies within the origin of both mating types. Phytophthora cinnamomi is the most invasive member of the genus with a host range of almost 5000 woody plant species [2,70,71]. Two genotypes of the A2 mating type have reached a panglobal distribution causing epidemics in numerous natural and managed ecosystems while the A1 mating type has a limited distribution outside of Asia and has never been associated with epidemic disease $[2,5,6,9-11,60,66,72-76]$.

Phytophthora attenuata, recently described from montane forests in Taiwan [22], and three closely related but previously unknown species, were found in the submontane and montane forests of northern Vietnam. Phytophthora attenuata, P. sp. attenuata-like 1 and P. sp. attenuata-like 2 were detected in the temperate, montane cloud forests around the Fansipan. However, P. sp. attenuata-like 3 was found only in the subtropical, humid submontane evergreen forests in Ba Vi NP. These four closely related species most likely result from sympatric species radiation, suggesting northern Indochina as the center of origin of the 'P. attenuata complex'. A pathogenicity trial is required to confirm that $P$. sp. attenuata-like 3 is causing the dieback of Dysoxylum juglans in Ba Vi National Park. Another Clade 7a species that was first described from Taiwan, $P$. $\times$ heterohybrida, was widespread in Vietnamese montane forest streams. This allopolyploid hybrid species has a functional but peculiar sexual system. In Taiwan, all isolates were selfsterile with both mating types being common and one isolate mating with both mating types [22]. In contrast, almost all Vietnamese isolates were self-sterile and belonged to the A1 mating type while one isolate was prolifically homothallic and stimulated oogonia formation in A2 tester strains.

The results indicate that the 'P. citricola complex' from Clade $2 c$ also underwent a species radiation process in Vietnam. Besides P. capensis, originally described from nursery plants in South Africa and also isolated from natural streams in Taiwan [10,77], and P. citricola VII, which was previously reported from a montane forest in Taiwan [10], four previously unknown taxa were found in this survey. Most common was P. citricola VII which occurred in mountain streams inside and outside of Hoàng Liên NP and in the rhizosphere of a montane Alnus forest, whereas the new taxa P. citricola VIII to XI had only cryptic distributions. It is noteable that P. citricola X and XI were only found in tropical lowland rainforests and that they differ from all other species of the 'P. citricola complex' by producing mainly papillate instead of semipapillate sporangia. The occurrence of $P$. citricola VII to X within only ca $300 \mathrm{~km}$ in northern Vietnam and the co-occurrence of P. citricola VII, VIII and IX in individual streams suggest sympatric species radiation from a common ancestor. In contrast, the exclusive finding of $P$. citricola XI and also $P$. sp. botryosa-like 2 from Clade 2a on Côn Lôn island, situated on the Asian shelf $50 \mathrm{~km}$ off the southern Vietnamese coast, are more consistent with allopatric island speciation. The invasive wide-host range pathogen P. plurivora occurs in undisturbed, healthy, often deciduous temperate mountain forests in Taiwan, Nepal and Yunnan [10,15,16,43]. However, it was not found here in the subtropical and tropical forests of Vietnam. This suggests that P. plurivora is native to temperate mountainous regions of South and East Asia.

The detection of five new species and of $P$. capensis from the ' $P$. citricola complex' in Clade $2 c$, three new species from Clade $2 \mathrm{a}$ and $P$. sp. tropicalis-like 2 and $P$. sp. multivesiculata-like 1 from Clades $2 \mathrm{~b}$ and $2 \mathrm{e}$ in this survey, the findings of P. bisheria, P. capensis, P. plurivora, P. citrophthora, P. tropicalis and the three new Clade 2a species $P$. sp. $\times$ botryosa-like, $P$. sp. $\times$ meadii-like and $P$. sp. occultans-like from natural ecosystems in Taiwan $[10,13]$ together with the widespread occurrence of P. botryosa, P. citricola, P. colocasiae and P. meadii across Southeast Asia [2,12,33-35] suggest South, Southeast and East Asia as the center of origin of Phytophthora major Clade 2.

Interestingly, all known isolates from the new Clade 2a taxa P. sp. meadii-like 1 and 2 and $P$. sp. botryosa-like 2 from Vietnam, as well as $P$. sp. $\times$ botryosa-like and $P$. sp. $\times$ meadii-like from Taiwan [10] are of A1 mating type and are characterised by oospore abortion rates exceeding 95\% in mating tests with tester strains of P. botryosa and P. meadii. It appears that in this complex of aerial Phytophthora species the A1 is better adapted to and, hence, more common in natural forests than the A2. It is even possible that these self-sterile taxa, like many aquatic Clade 6 species [21,53], lack the A2 mating 
type and have abandoned sexual reproduction in favour of exclusive asexual reproduction, spreading via their caducous sporangia from infected to non-infected above-ground tissues. This possibility is supported by the extremely high oospore abortion rates in mating tests with tester strains of $P$. botryosa and P. meadii. More field surveys and laboratory tests are needed to verify this hypothesis.

Phytophthora castaneae and P. heveae from Clade 5 are also considered being native to Taiwan and Hainan $[2,10,12,31]$. Their widespread occurrence in Vietnamese forests and the lack of association with disease symptoms in the native vegetation indicate that Indochina also lies within the origin of both species.

As previously demonstrated in Australia, Chile, South Africa and Taiwan putative interspecific hybrids, indicated by multiple heterozygous sites in their ITS sequences, are common in watercourses and can also be found in forest soils $[10,11,17,18,78,79]$. As with predominantly aquatic species and hybrids from Clade 6, all Clade 9 hybrids from Vietnamese streams, with the exception of $P$. sp. $\times$ insolita-like which produces oogonia without antheridia, are sterile and apparently adapted to rapid and continuous asexual proliferation via zoospores. Also, like many Clade 6 taxa, this may reflect adaptation to a mostly saprotrophic lifestyle as decomposers of naturally fallen leaves [21,53]. As with the Clade 6 hybrid P. thermophila $\times$ P. amnicola in the Valdivia River in Chile [11], no putative parents of the Clade 9 hybrids $P$. sp. $\times$ Grenada 3-like, $P$. sp. $\times$ insolita-like, $P$. sp. $\times$ kunnunara-like, $P$. sp. $\times$ Peru 4-like and $P$. sp. $\times$ virginiana-like were detected in the Vietnamese rivers. Possibly the hybridisation events occurred in the Vietnamese streams and the parents were outcompeted by the better adapted hybrids. Alternatively, the hybrids could be introduced from elsewhere. Since the multicopy ITS locus is of limited use for hybrid studies sequencing of appropriate mitochondrial and nuclear genes are needed to confirm the hybrid status and elucidate the parents of the putative hybrid taxa.

Panglobally distributed pathogens from Phytophthora Clades 1 (P. cactorum, P. infestans, P. nicotianae) and 4 (P. palmivora) commonly cause diseases of horticultural crops and ornamental plants in mainland China, Hainan and Taiwan $[12,33,35]$. However, in this survey, as in previous surveys in Taiwan, species from Clades 1 and 4 (exception for one isolate of P. palmivora in Taiwan) were not detected in natural forests and streams $[10,13]$, indicating that these two clades are not native to Taiwan and Southeast Asia. The same probably applies to Clades 3, 11 and 12 [23,28].

Although the natural hosts of the putatively endemic Vietnamese forest Phytophthoras obtained in this study are still unknown, it is evident that many native Asian forest Phytophthoras have co-evolved with a variety of tree genera also present in Europe and North America, including Fagaceae, Lauraceae, Aceraceae, Oleaceae, and Pinaceae. In this case high susceptibility of many non-coevolved European and North American trees to these Asian Phytophthora species is possible, as already well demonstrated for P. cinnamomi, P. plurivora, P. Xcambivora and, more recently, for six new Clade 7a species from Taiwan [22]. An extensive host range study with Phytophthora species from Asia, South and Central America has been initiated and will be published separately. In one part of this study, the pathogenicity of five Asian species (P. castaneae, P. heveae and the three new Vietnamese species P. citricola X, P. sp. multivesiculata-like 1 and P. sp. tropicalis-like 2) to Castanea sativa, Quercus suber and Quercus robur has been investigated and all five caused significant rot and loss of fine roots and suberised lateral roots in all three hosts, C. sativa being most susceptible [80].

Against this background, the annual importation of over three billion plants-for-planting into Europe [81], the large numbers of previously unknown Phytophthora species in natural and horticultural ecosystems being identified in Asia, South and Central America ([10,11,16,22,82], this study) and the occurrence of at least 47 exotic Phytophthora species in European nurseries and associated outplantings [60] represents a significant biosecurity risk for forestry, horticulture, and natural ecosystems in Europe and North America.

Many recent epidemics of trees and horticultural crops have been caused by introduced pathogens that were previously unknown to science, probably due to the organisms being co-evolved and benign in their centres of origin $[6,10,83]$. Although often introduced via the plants-for-planting pathway, none of them has ever been intercepted pre-emptively during routine phytosanitary controls 
at the ports of entry $[60,81,83,84]$. Despite overwhelming scientific evidence, current sanitary and phytosanitary (SPS) protocols largely ignore the risks from unknown, benign, co-evolved and unescaped organisms [6,60,83-85]. However, preventing further introductions of potentially harmful invasive Phytophthoras is a key issue for international forest biosecurity. A series of international research projects and organisations (listed in [10]) have come to similar conclusions. The current, outdated and scientifically flawed species-by-species regulation approach based on random visual inspections for symptoms of described pests and pathogens needs to be replaced by a sophisticated pathway regulation approach using pathway risk analyses, risk-based inspection regimes and molecular high-throughput detection tools $[6,60,81,83,84,86,87]$.

To further define areas of Phytophthora diversity, including high-risk areas for the origin of potentially harmful pathogens, more Phytophthora surveys are needed in natural ecosystems in unsurveyed areas of Asia, Africa, and South and Central America, followed by host range testing of new taxa on naive tree hosts in Europe and elsewhere. Such surveys should also contribute to a better understanding of the global diversity of Phytophthora, the ancient biogeographic radiation of the Phytophthora species and Clades, and the influence of local environmental and host factors on breeding strategies and adaptation in the genus.

\section{Conclusions}

A remarkable diversity of 13 described species, five informally designated taxa and 21 previously unknown taxa of Phytophthora were obtained from 25 natural and semi-natural forest stands and 16 rivers in temperate and subtropical montane and tropical lowland regions of Vietnam. It is concluded that Vietnam is within the center of origin of most Phytophthora taxa found, including P. cinnamomi and P. ramorum, and that Phytophthora clades 2, 5, 6, 7, 8, 9, and 10 are native to Indochina.

Supplementary Materials: The following are available online at http://www.mdpi.com/1999-4907/11/1/93/s1, Table S1: GenBank accession numbers of ITS and partial cox1 sequences generated in this study for representative Phytophthora, Elongisporangium, Nothophytophthora, Phytopythium and Pythium isolates from Vietnamese forests and rivers and isolates from related Phytophthora species used for comparisons.

Author Contributions: Conceptualization: T.J., M.H.J., C.M.B.; data curation: T.J., M.H.J., I.M.; formal analysis: T.J., M.H.J., B.S., M.T., M.P., J.B., H.R., A.P.-S., A.B., M.R., C.M.; investigation: T.J., M.H.J., C.M.B., J.W., T.C., B.S.; methodology: T.J., M.H.J., C.M.B.; writing-original draft: T.J.; writing-review and editing: T.J., C.M.B., B.S., T.C., I.M., M.T., J.B., M.H.J., A.P.-S. All authors have read and agree to the published version of the manuscript.

Funding: The authors are grateful to the Czech Ministry for Education, Youth and Sports and the European Regional Development Fund for financing the Project Phytophthora Research Centre Reg. No. CZ.02.1.01/0.0/0.0/15_003/0000453. This work has also received funding from the European Union's Horizon 2020 research and innovation programme under grant agreement No. 635646, POnTE (Pest Organisms Threatening Europe). Travel and subsistence support for CMB was provided by Brasier Consultancy. DNA sequencing in this study was partly supported by the Hungarian Scientific Research Fund (OTKA) grant K101914.

Acknowledgments: The Vietnamese Academy of Forest Sciences in Hanoi is gratefully acknowledged for providing lab space and technical support. We thank Henrieta Ďatková and Josef Janoušek (PRC, Brno, Czech Republic) for much appreciated technical support.

Conflicts of Interest: The authors declare no conflict of interest.

\section{References}

1. Brasier, C.M.; Robredo, F.; Ferraz, J.F.P. Evidence for Phytophthora cinnamomi involvement in Iberian oak decline. Plant Pathol. 1993, 42, 140-145. [CrossRef]

2. Erwin, D.C.; Ribeiro, O.K. Phytophthora Diseases Worldwide; APS Press: Saint Paul, MN, USA, 1996.

3. Hansen, E.M.; Goheen, D.J.; Jules, E.S.; Ullian, B. Managing Port-Orford-Cedar and the introduced pathogen Phytophthora lateralis. Plant Dis. 2000, 84, 4-14. [CrossRef] [PubMed]

4. Jung, T.; Blaschke, H.; Osswald, W. Involvement of soilborne Phytophthora species in Central European oak decline and the effect of site factors on the disease. Plant Pathol. 2000, 49, 706-718. [CrossRef] 
5. Jung, T.; Vettraino, A.M.; Cech, T.L.; Vannini, A. The impact of invasive Phytophthora species on European forests. Phytophthora: A Global Perspective; Lamour, K., Ed.; CABI: Wallingford, UK, 2013; pp. 146-158, ISBN 978-1-78064-093-8.

6. Jung, T.; Pérez-Sierra, A.; Durán, A.; Horta Jung, M.; Balci, Y.; Scanu, B. Canker and decline diseases caused by soil- and airborne Phytophthora species in forests and woodlands. Persoonia 2018, 40, 182-220. [CrossRef]

7. Rizzo, D.M.; Garbelotto, M.; Davidson, J.M.; Slaugter, G.W. Phytophthora ramorum as the cause of extensive mortality of Quercus spp. and Lithocarpus densiflorus in California. Plant Dis. 2002, 86, 205-214. [CrossRef]

8. Hardham, A.R. Phytophthora cinnamomi. Mol. Plant Pathol. 2005, 6, 589-604. [CrossRef]

9. Cahill, D.M.; Rookes, J.E.; Wilson, B.A.; Gibson, L.; Mcdougall, K.L. Turner Review No. 17. Phytophthora cinnamomi and Australia's biodiversity: Impacts, predictions and progress towards control. Aust. J. Bot. 2008, 56, 279-310. [CrossRef]

10. Jung, T.; Chang, T.T.; Bakonyi, J.; Seress, D.; Pérez-Sierra, A.; Yang, X.; Hong, C.; Scanu, B.; Fu, C.H.; Hsueh, K.-L.; et al. Diversity of Phytophthora species in natural ecosystems of Taiwan and association with disease symptoms. Plant Pathol. 2017, 66, 194-211. [CrossRef]

11. Jung, T.; Durán, A.; Sanfuentes von Stowasser, E.; Schena, L.; Mosca, S.; Fajardo, S.; González, M.; Navarro Ortega, A.D.; Bakonyi, J.; Seress, D.; et al. Diversity of Phytophthora species in Valdivian rainforests and association with severe dieback symptoms. Forest Pathol. 2018, 48, e12443. [CrossRef]

12. Zeng, H.-C.; Ho, H.-H.; Zheng, F.-C. A survey of Phytophthora species on Hainan Island of South China. J. Phytopathol. 2009, 157, 33-39. [CrossRef]

13. Brasier, C.M.; Vettraino, A.M.; Chang, T.T.; Vannini, A. Phytophthora lateralis discovered in an old growth Chamaecyparis forest in Taiwan. Plant Pathol. 2010, 59, 595-603. [CrossRef]

14. Reeser, P.W.; Sutton, W.; Hansen, E.M.; Remigi, P.; Adams, G.C. Phytophthora species in forest streams in Oregon and Alaska. Mycologia 2011, 103, 22-35. [CrossRef] [PubMed]

15. Vettraino, A.M.; Brasier, C.M.; Brown, A.V.; Vannini, A. Phytophthora himalsilva sp. nov. an unusually phenotypically variable species from a remote forest in Nepal. Fungal Biol. 2011, 115, 275-287. [CrossRef] [PubMed]

16. Huai, W.X.; Tian, G.; Hansen, E.M.; Zhao, W.X.; Goheen, E.M.; Grünwald, N.J.; Cheng, C. Identification of Phytophthora species baited and isolated from forest soil and streams in northwestern Yunnan province, China. Forest Pathol. 2013, 43, 87-103. [CrossRef]

17. Hüberli, D.; Hardy, G.E.S.T.J.; White, D.; Williams, N.; Burgess, T.I. Fishing for Phytophthora from Western Australia' s waterways: A distribution and diversity survey. Australas. Plant Pathol. 2013, 42, 251-260. [CrossRef]

18. Oh, E.; Gryzenhout, M.; Wingfield, B.D.; Wingfield, M.J.; Burgess, T.I. Surveys of soil and water reveal a goldmine of Phytophthora diversity in South African natural ecosystems. IMA Fungus 2013, 4, 123-131. [CrossRef] [PubMed]

19. Burgess, T.I.; White, D.; McDougall, K.M.; Garnas, J.; Dunstan, W.A.; Català, S.; Carnegie, A.J.; Worboys, S.; Cahill, D.; Vettraino, A.M.; et al. Distribution and diversity of Phytophthora across Australia. Pac. Conserv. Biol. 2017, 23, 1-13. [CrossRef]

20. Jung, T.; Hansen, E.M.; Winton, L.; Oßwald, W.; Delatour, C. Three new species of Phytophthora from European oak forests. Mycol. Res. 2002, 106, 397-411. [CrossRef]

21. Jung, T.; Stukely, M.J.C.; Hardy, G.E.S.J.; White, D.; Paap, T.; Dunstan, W.A.; Burgess, T.I. Multiple new Phytophthora species from ITS Clade 6 associated with natural ecosystems in Australia: Evolutionary and ecological implications. Persoonia 2011, 26, 13-39. [CrossRef]

22. Jung, T.; Horta Jung, M.; Scanu, B.; Seress, D.; Kovács, D.M.; Maia, C.; Pérez-Sierra, A.; Chang, T.-T.; Chandelier, A.; Heungens, A.; et al. Six new Phytophthora species from ITS Clade 7a including two sexually functional heterothallic hybrid species detected in natural ecosystems in Taiwan. Persoonia 2017, 38, 100-135. [CrossRef]

23. Jung, T.; Horta Jung, M.; Cacciola, S.O.; Cech, T.; Bakonyi, J.; Seress, D.; Mosca, S.; Schena, L.; Seddaiu, S.; Pane, A.; et al. Multiple new cryptic pathogenic Phytophthora species from Fagaceae forests in Austria, Italy and Portugal. IMA Fungus 2017, 8, 219-244. [CrossRef] [PubMed]

24. Burgess, T.I.; Webster, J.L.; Ciampini, J.A.; White, D.; Hardy, G.E.S.J.; Stukely, M.J.C. Re-evaluation of Phytophthora species isolated during 30 years of vegetation health surveys in Western Australia using molecular techniques. Plant Dis. 2009, 93, 215-223. [CrossRef] [PubMed] 
25. Burgess, T.I.; Simamora, A.V.; White, D.; Wiliams, B.; Schwager, M.; Stukely, M.J.C.; Hardy, G.E.S.J. New species from Phytophthora Clade 6a: Evidence for recent radiation. Persoonia 2018, 41, 1-7. [CrossRef] [PubMed]

26. Brasier, C. Phytophthora biodiversity: How many Phytophthora species are there? In Phytophthoras in Forests and Natural Ecosystems: Fourth Meeting of the International Union of Forest Research Organizations (IUFRO) Working Party S07.02.09, USDA Forest Service; Goheen, E.M., Frankel, S.J., Eds.; Pacific Southwest Research Station: Albany, NY, USA, 2009.

27. Kroon, L.P.; Brouwer, H.; de Cock, A.W.; Govers, F. The genus Phytophthora anno 2012. Phytopathology 2012, 102, 348-364. [CrossRef] [PubMed]

28. Yang, X.; Tyler, B.M.; Hong, C. An expanded phylogeny for the genus Phytophthora. IMA Fungus 2017, 8, 355-384. [CrossRef]

29. Cooke, D.E.L.; Drenth, A.; Duncan, J.M.; Wagels, G.; Brasier, C.M. A molecular phylogeny of Phytophthora and related Oomycetes. Fungal Genet. Biol. 2000, 30, 17-32. [CrossRef]

30. Ko, W.H.; Chang, H.S.; Su, H.J. Isolates from Phytophthora cinnamomi from Taiwan as evidence for an Asian origin of the species. Trans. Br. Mycol. Soc. 1978, 71, 496-499. [CrossRef]

31. Ko, W.H.; Wang, S.Y.; Ann, P.J. The possible origin and relation of Phytophthora katsurae and P. heveae, discovered in a protected natural forest in Taiwan. Bot. Stud. 2006, 47, 273-277.

32. Chang, T.T.; Wang, W.W.; Wang, W.Y. Use of random amplified polymorphic DNA markers for the detection of genetic variation in Phytophthora cinnamomi in Taiwan. Bot. Bull. Acad. Sin. 1996, 37, 165-171. Available online: https://ejournal.sinica.edu.tw/bbas/content/1996/3/bot373-01.html (accessed on 16 December 2019).

33. Ho, H.H.; Lu, J.Y. A synopsis of the occurrence and pathogenicity of Phytophthora species in mainland China. Mycopathologia 1997, 138, 143-161. [CrossRef]

34. Drenth, A.; Guest, D.I. Diversity and management of Phytophthora in Southeast Asia; ACIAR Monograph 114; Australian Centre for International Agricultural Research: Canberra, Australia, 2004; p. 238, ISBN 1-86320-405-9.

35. Ann, P.J.; Wong, I.T.; Tsai, J.N. New records of Phytophthora diseases of aromatic crops in Taiwan. Plant Pathol. Bull. 2010, 19, 53-68.

36. Chang-Fu, H.; Chung-Fu, S. Introduction to the flora of Taiwan, 1: Geography, Geology, Climate, and Soils. Flora of Taiwan Second Edition. Editorial Committee of the Flora of Taiwan Second Edition. 1994. Available online: http://tai2.ntu.edu.tw/ebook/ebookpage.php?volume=1\&book=Fl.\%20Taiwan\%202nd\% 20edit.\&page $=1$ (accessed on 11 January 2020).

37. Chang-Fu,H.; Chung-Fu, S.; Kuoh-Cheng, Y. Introduction to the Flora of Taiwan, 3: Floristics, Phytogeography, and Vegetation. Flora of Taiwan Second Edition. Editorial Committee of the Flora of Taiwan Second Edition. 1994. Available online: http://tai2.ntu.edu.tw/ebook/ebookpage.php?book=Fl.\%20Taiwan\%202nd\%20edit. \&volume $=1$ \&page $=7$ (accessed on 11 January 2020).

38. Chung-Fu, S. Introduction to the flora of Taiwan, 2: Geotectonic Evolution, Paleogeography, and the Origin of the Flora. Flora of Taiwan Second Edition. Editorial Committee of the Flora of Taiwan Second Edition. 1994. Available online: http://tai2.ntu.edu.tw/ebook/ebookpage.php?book=Fl.\%20Taiwan\%202nd\%20edit. \&volume $=1 \&$ page $=3$ (accessed on 11 January 2020).

39. Gower, D.J.; Johnson, K.G.; Richardson, J.E.; Rosen, B.R.; Rüber, L.; Williams, S.T. Biotic Evolution and Environmental Change in Southeast Asia; The Systematics Association Special Volume 82; Cambridge University Press: New York, NY, USA, 2012; ISBN 13- 978-1107001305.

40. Averyanov, L.V.; Loc, P.K.; Hiep, N.T.; Harder, D.K. Phytogeographic review of Vietnam and adjacent areas of Eastern Indochina. Komarovia 2003, 3, 1-83.

41. Wurster, C.M.; Bird, M.I.; Bull, I.D.; Creed, F.; Bryant, C.; Dungait, J.A.J.; Paz, V. Forest contraction in north equatorial Southeast Asia during the last glacial maximum. Proc. Natl. Acad. Sci. USA 2010, 107, 15508-15511. [CrossRef] [PubMed]

42. Jung, T. Beech decline in Central Europe driven by the interaction between Phytophthora infections and climatic extremes. Forest Pathol. 2009, 39, 73-94. [CrossRef]

43. Jung, T.; Burgess, T.I. Re-evaluation of Phytophthora citricola isolates from multiple woody hosts in Europe and North America reveals a new species, Phytophthora plurivora sp. nov. Persoonia 2009, 22, 95-110. [CrossRef]

44. Hansen, E.M.; Reeser, P.W.; Sutton, W. Phytophthora beyond agriculture. Annu. Rev. Phytopathol. 2012, 50, 359-378. [CrossRef] 
45. Scanu, B.; Hunter, G.C.; Linaldeddu, B.T.; Franceschini, A.; Maddau, L.; Jung, T.; Denman, S. A taxonomic re-evaluation reveals that Phytophthora cinnamomi and P. cinnamomi var. parvispora are separate species. Forest Pathol. 2014, 44, 1-20. [CrossRef]

46. White, T.J.; Bruns, T.; Lee, S.; Taylor, J. Amplification and direct sequencing of fungal ribosomal RNA genes for phylogenetics. In PCR Protocols: A Guide to Methods and Applications; Innis, M.A., Gelfand, D.H., Sninsky, J.J., White, T.J., Eds.; Academic Press: San Diego, CA, USA, 1990; pp. 315-322, ISBN 0123721806.

47. Kroon, L.P.N.M.; Bakker, F.T.; van den Bosch, G.B.M.; Bonants, P.J.M.; Flier, W.G. Phylogenetic analysis of Phytophthora species based on mitochondrial and nuclear DNA sequences. Fungal Genet. Biol. 2004, 41, 766-782. [CrossRef]

48. Martin, F.N.; Tooley, P.W. Phylogenetic relationships among Phytophthora species inferred from sequence analysis of mitochondrially encoded cytochrome oxidase I and II genes. Mycologia 2003, 95, 269-284. [CrossRef]

49. Aragaki, M.; Uchida, J.Y. Morphological distinctions between Phytophthora capsici and P. tropicalis sp. nov. Mycologia 2001, 93, 137-145. [CrossRef]

50. Ilieva, E.; Man In't Veld, W.A.; Veenbaas-Rijks, W.; Pieters, R. Phytophthora multivesiculata, a new species causing rot in Cymbidium. Eur. J. Plant Pathol. 1998, 104, 677-684. [CrossRef]

51. Jung, T.; Scanu, B.; Bakonyi, J.; Seress, D.; Kovács, G.M.; Durán, A.; Sanfuentes von Stowasser, E.; Schena, L.; Mosca, S.; Thu, P.Q.; et al. Nothophytophthora gen. nov., a new sister genus of Phytophthora from natural and semi-natural ecosystems. Persoonia 2017, 39, 143-174. [CrossRef]

52. Werres, S.; Marwitz, R.; Man In't Veld, W.A.M.; Bonants, P.J.M.; De Weerd, M.; Themann, K.; Ilieva, E.; Baayen, R.P. Phytophthora ramorum sp. nov., a new pathogen on Rhododendron and Viburnum. Mycol. Res. 2001, 105, 1155-1165. [CrossRef]

53. Brasier, C.M.; Cooke, D.E.L.; Duncan, J.M.; Hansen, E.M. Multiple new phenotypic taxa from trees and riparian ecosystems in Phytophthora gonapodyides-P. megasperma ITS Clade 6, which tend to be high-temperature tolerant and either inbreeding or sterile. Mycol. Res. 2003, 107, 277-290. [CrossRef]

54. Safaiefarahani, B.; Mostowfizadeh-Ghalamfarsa, R.; Hardy, G.E.S.J.; Burgess, T.I. Re-evaluation of the Phytophthora cryptogea species complex and the description of a new species, Phytophthora pseudocryptogea sp. nov. Mycol. Prog. 2015, 14, 108. [CrossRef]

55. Jung, T.; Nechwatal, J. Phytophthora gallica sp. nov., a new species from rhizosphere soil of declining oak and reed stands in France and Germany. Mycol. Res. 2008, 112, 1195-1205. [CrossRef]

56. Yang, X.; Hong, C. Phytophthora virginiana sp. nov., a high-temperature tolerant species from irrigation water in Virginia. Mycotaxon 2013, 126, 167-176. [CrossRef]

57. Balci, Y.; Balci, S.; Eggers, J.; MacDonald, W.L.; Juzwik, J.; Long, R.P.; Gottschalk, K.W. Phytophthora spp. associated with forest soils in eastern and north-central U.S. oak ecosystems. Plant Dis. 2007, 91, 705-710. [CrossRef]

58. Jung, T.; La Spada, F.; Pane, A.; Aloi, F.; Evoli, M.; Horta Jung, M.; Scanu, B.; Faedda, R.; Rizza, C.; Puglisi, I.; et al. Diversity and distribution of Phytophthora species in protected natural areas in Sicily. Forests 2019, 10, 259. [CrossRef]

59. Milenković, I.; Keča, N.; Karadžić, D.; Radulović, Z.; Nowakowska, J.A.; Oszako, T.; Sikora, K.; Corcobado, T.; Jung, T. Isolation and pathogenicity of Phytophthora species from poplar plantations in Serbia. Forests 2018, 9, 330. [CrossRef]

60. Jung, T.; Orlikowski, L.; Henricot, B.; Abad-Campos, P.; Aday, A.G.; Aguín Casal, O.; Bakonyi, J.; Cacciola, S.O.; Cech, T.; Chavarriaga, D.; et al. Widespread Phytophthora infestations in European nurseries put forest, semi-natural and horticultural ecosystems at high risk of Phytophthora diseases. For. Pathol. 2016, 46, 134-163. [CrossRef]

61. Shrestha, S.K.; Zhou, Y.; Lamour, K. Oomycetes baited from streams in Tennessee 2010-2012. Mycologia 2013, 105, 1516-1523. [CrossRef]

62. Brasier, C.M. Evolutionary Biology of Phytophthora. I. Genetic system, sexuality and variation. Annu. Rev. Phytopathol. 1992, 30, 153-171. [CrossRef]

63. Català, S.; Peréz-Sierra, A.; Abad-Campos, P. The use of genus-specific amplicon pyrosequencing to assess Phytophthora species diversity using eDNA from soil and water in Northern Spain. PLoS ONE 2015, 10, e0119311. [CrossRef] 
64. Grünwald, N.J.; Garbelotto, M.; Goss, E.M.; Heungens, K.; Prospero, S. Emergence of the sudden oak death pathogen Phytophthora ramorum. Trends Microbiol. 2012, 20, 131-138. [CrossRef]

65. Van Poucke, K.; Franceschini, S.; Webber, J.F.; Vercauteren, A.; Turner, J.A.; McCracken, A.R.; Heungens, K.; Brasier, C.M. Discovery of a fourth evolutionary lineage of Phytophthora ramorum: EU2. Fungal Biol. 2012, 116, 1178-1191. [CrossRef]

66. Arentz, F.; Simpson, J.A. Distribution of Phytophthora cinnamomi in Papua New Guinea and notes on its origin. Trans. Br. Mycol. Soc. 1986, 87, 289-295. [CrossRef]

67. Brasier, C.M.; Scott, J.K. European oak declines and global warming: A theoretical assessment with special reference to the activity of Phytophthora cinnamomi. Bull. OEPP EPPO Bull. 1994, 24, 221-234. [CrossRef]

68. Brasier, C.M. Phytophthora cinnamomi and oak decline in southern Europe. Environmental constraints including climate change. Ann. Des. Sci. For. 1996, 53, 347-358. [CrossRef]

69. Burgess, T.I.; Scott, J.K.; McDougall, K.L.; Stukely, M.J.C.; Crane, C.; Dunstan, W.A.; Brigg, F.; Andjic, V.; White, D.; Rudman, T.; et al. Current and projected global distribution of Phytophthora cinnamomi, one of the world's worst plant pathogens. Glob. Chang. Biol. 2017, 23, 1661-1674. [CrossRef]

70. Shearer, B.L.; Crane, C.E.; Cochrane, A. Quantification of the susceptibility of the native flora of the South-West Botanical Province, Western Australia, to Phytophthora cinnamomi. Aust. J. Bot. 2004, 52, 435-443. [CrossRef]

71. Hardham, A.R.; Blackman, L.M. Phytophthora cinnamomi. Mol. Plant Pathol. 2018, 19, 260-285. [CrossRef] [PubMed]

72. Zentmyer, G.A. Phytophthora Cinnamomi and the Diseases it Causes; Monograph No. 10; The American Phytopathological Society: Saint Paul, MN, USA, 1980; ISBN 0890540306.

73. Linde, C.; Drenth, A.; Kemp, G.H.J.; Wingfield, M.J.; Von Broembsen, S.L. Population structure of Phytophthora cinnamomi in South Africa. Phytopathology 1997, 87, 822-827. [CrossRef] [PubMed]

74. Dobrowolski, M.P.; Tommerup, I.C.; Blakeman, H.D.; O’Brien, P.A. Non-Mendelian inheritance revealed in a genetic analysis of sexual progeny of Phytophthora cinnamomi with microsatellite markers. Fungal Genet. Biol. 2002, 35, 197-212. [CrossRef] [PubMed]

75. Dobrowolski, M.P.; Tommerup, I.C.; Shearer, B.L.; O'Brien, P.A. Three clonal lineages of Phytophthora cinnamomi in Australia revealed by microsatellites. Phytopathology 2003, 93, 695-704. [CrossRef] [PubMed]

76. Jung, T.; Colquhoun, I.J.; Hardy, G.E.S.J. New insights into the survival strategy of the invasive soilborne pathogen Phytophthora cinnamomi in different natural ecosystems in Western Australia. Forest Pathol. 2013, 43, 266-288. [CrossRef]

77. Bezuidenhout, C.M.; Denman, S.; Kirk, S.A.; Botha, W.J.; Mostert, L.; McLeod, A. Phytophthora taxa associated with cultivated Agathosma, with emphasis on the P. citricola complex and P. capensis sp. nov. Persoonia 2010, 25, 32-49. [CrossRef]

78. Nagel, J.H.; Gryzenhout, M.; Slippers, B.; Wingfield, M.J.; Hardy, G.E.S.J.; Stukely, M.; Burgess, T.I. Characterization of Phytophthora hybrids from ITS clade 6 associated with riparian ecosystems in South Africa and Australia. Fungal Biol. 2013, 117, 329-347. [CrossRef]

79. Burgess, T.I. Molecular characterization of natural hybrids formed between five related indigenous Clade 6 Phytophthora species. PLoS ONE 2015, 10, e0134225. [CrossRef]

80. Jung, T.; Maia, C.; Horta Jung, M. Host range testing of known and novel Phytophthora species from Asia, Central and South America among major forest tree species from Europe. Unpublished work. 2020.

81. Eschen, R.; Douma, J.C.; Grégoire, J.-C.; Mayer, F.; Rigaux, L.; Potting, R.P.J. A risk categorisation and analysis of the geographic and temporal dynamics of the European import of plants for planting. Biol. Invasions 2017, 19, 3243-3257. [CrossRef]

82. Rahman, M.Z.; Uematsu, S.; Takeuchi, T.; Shirai, K.; Ishiguro, Y.; Suga, H.; Kageyama, K. Two new species, Phytophthora nagaii sp. nov. and P. fragariaefolia sp. nov., causing serious diseases on rose and strawberry plants, respectively, in Japan. J. Gen. Plant Pathol. 2014, 80, 348-365. [CrossRef]

83. Brasier, C.M. The biosecurity threat to the UK and global environment from international trade in plants. Plant Pathol. 2008, 57, 792-808. [CrossRef]

84. Santini, A.; Ghelardini, L.; De Pace, C.; Desprez-Loustau, M.L.; Capretti, P.; Chandelier, A.; Cech, T.; Chira, D.; Diamandis, S.; Gaitniekis, T.; et al. Biogeographic patterns and determinants of invasion by alien forest pathogens in Europe. New Phytol. 2013, 197, 238-250. [CrossRef] [PubMed]

85. Liebhold, A.M.; Brockerhoff, E.G.; Garrett, L.J.; Parke, J.L.; Britton, K.O. Live plant imports: The major pathway for forest insect and pathogen invasions of the US. Front. Ecol. Environ. 2012, 10, 135-143. [CrossRef] 
86. Eschen, R.; Rigaux, L.; Sukovata, L.; Vettraino, A.M.; Marzano, M.; Grégoire, J.-C. Phytosanitary inspection of woody plants for planting at European Union entry points: A practical enquiry. Biol. Invasions 2015, 17, 2403-2413. [CrossRef]

87. Eschen, R.; Britton, K.; Brockerhoff, E.; Burgess, T.; Dalley, V.; Epanchin-Niell, R.; Gupta, K.; Hardy, G.; Huang, Y.; Kenis, M.; et al. International variation in phytosanitary legislation and regulations governing importation of plants for planting. Environ. Sci. Policy 2015, 51, 228-237. [CrossRef]

(C) 2020 by the authors. Licensee MDPI, Basel, Switzerland. This article is an open access article distributed under the terms and conditions of the Creative Commons Attribution (CC BY) license (http://creativecommons.org/licenses/by/4.0/). 